\title{
Primordial Magnetic Field Limits from CMB Trispectrum - Scalar Modes and Planck Constraints
}

\author{
Pranjal Trivedi \\ Department of Physics, Sri Venkateswara College, University of Delhi, Delhi 110021, India, \\ \& Department of Physics and Astrophysics, University of Delhi, Delhi 110007, India * \\ Kandaswamy Subramanian \\ IUCAA, Post Bag 4, Ganeshkhind, Pune 411 007, India \\ T. R. Seshadri \\ Department of Physics and Astrophysics, University of Delhi, Delhi 110007, India
}

(Dated: October 12, 2018)

\begin{abstract}
Cosmic magnetic fields are observed to be coherent on large scales and could have a primordial origin. NonGaussian signals in the cosmic microwave background (CMB) are generated by primordial magnetic fields as the magnetic stresses and temperature anisotropy they induce depend quadratically on the magnetic field. We compute the CMB scalar trispectrum on large angular scales, for nearly scale-invariant magnetic fields, sourced via the Sachs-Wolfe effect. The trispectra induced by magnetic energy density and by magnetic scalar anisotropic stress are found to have typical magnitudes of approximately $10^{-29}$ and $10^{-19}$, respectively. The scalar anisotropic stress trispectrum is also calculated in the flat-sky approximation and yields a similar result. Observational limits on CMB non-Gaussianity from the Planck mission data allow us to set upper limits of $B_{0} \lesssim$ $0.6 \mathrm{nG}$ on the present value of the primordial cosmic magnetic field. Considering the inflationary magnetic curvature mode in the trispectrum can further tighten the magnetic field upper limit to $B_{0} \lesssim 0.05 \mathrm{nG}$. These sub-nanoGauss constraints from the magnetic trispectrum are the most stringent limits so far on the strength of primordial magnetic fields, on megaparsec scales, significantly better than the limits obtained from the CMB bispectrum and the $\mathrm{CMB}$ power spectrum.
\end{abstract}

\section{INTRODUCTION}

Magnetic fields have been observed throughout the Universe, on all scales probed so far, from planets and stars to the large-scale magnetic fields detected in galaxies and galaxy clusters [1-8]. Both large-scale as well as stochastic components are present in magnetic fields observed in galaxies with magnitudes from a few to tens of microGauss. Coherent magnetic fields of a similar strength are also observed in higher redshift galaxies [9, 10]. In clusters of galaxies, stochastic magnetic fields of a few microGauss strength are present, correlated on ten kiloparsec scales [3, 4]. Moreover, there is circumstantial evidence of an intergalactic magnetic field that is present over most of the cosmic volume,even in the voids of large scale structure. A lower bound of $10^{-16}-10^{-15}$ Gauss for such a pervasive intergalactic magnetic field has been derived from gamma-ray observations of blazars $[11-13]$.

The origin as well as evolution of such large-scale magnetic fields remains an outstanding problem. Magnetic fields in collapsed structures can arise from dynamo amplification of seed magnetic fields $[6-8]$. The seed field could in turn be generated in astrophysical batteries [14-17] or due to processes in the early universe [18-28]. Indeed, the recent gamma-ray observations suggesting a lower limit to an all-pervasive intergalactic magnetic field [11-13], would perhaps favour a primordial origin. A primordial magnetic field can be generated at inflation [5, 18 -23], or arise out of other phase tran-

\footnotetext{
* ptrivedi@physics.du.ac.in

$\dagger$ kandu@iucaa.ernet.in

$\ddagger$ trs@physics.du.ac.in
}

sitions in the early Universe [24-28]. As yet there is no compelling mechanism which produces strong coherent primordial fields. Equally, the dynamo paradigm is not without its own challenges in producing sufficiently coherent fields and sufficiently rapidly [6-8]. Therefore, it is useful to keep open the possibility that primordial magnetic fields originating in the early universe play a crucial role in explaining the observed cosmic magnetism.

In this context it is important to investigate every possible observable signature of the putative primordial magnetic field. Magnetic fields give rise to scalar, vector and tensor metric perturbations as well as fluid perturbations via the Lorentz force. Constraints on large scale primordial magnetic fields have already been derived using the CMB temperature and polarization power spectra [29-36] and Faraday rotation [37[39]. However, the effects of a primordial magnetic field on the CMB are relatively more pronounced in its non-Gaussian correlations. This arises due to the fact that magnetic fields induce non-Gaussian signals at lowest order as the magnetic energy density and stress are quadratic in the field. In contrast, the standard inflationary perturbations, dominated by their linear component, can source non-Gaussian correlations only with higher order perturbations and thus necessarily can only produce a small amplitude of CMB non-Gaussianity (cf. [40-48]). Primordial magnetic fields have been shown capable of inducing appreciable CMB non-Gaussianity when considering the bispectrum [49-59]. Our earlier calculation of the magnetic CMB bispectrum sourced by scalar anisotropic stress led to $\mathrm{a} \sim 2 \mathrm{nG}$ upper limit on the primordial magnetic field's amplitude on megaparsec scales [59]. However, higherorder measures of non-Gaussianity like the trispectrum have been less investigated and as we show here, are very useful to 
set further constraints on primordial magnetic fields.

In this article we present in detail the primordial magnetic field contribution to the CMB scalar mode trispectrum. The principal results were summarized in our earlier Letter [60], where WMAP5 and WMAP7 constraints on nonGaussianity were used to derive magnetic field constraints. Here we present the full trispectrum calculations as well as an additional flat-sky calculation for the scalar anisotropic stress trispectrum. Furthermore, the new constraints on nonGaussianity from the Planck mission 2013 data release [61] are utilized to obtain improved magnetic field constraints. We find that the trispectrum does better than the bispectrum at probing magnetic fields on large scales.We also show that even stronger constraints can be imposed on magnetic fields by considering the recently discussed magnetic inflationary curvature mode [62].

In the next section we describe the properties of the stochastic primordial magnetic field assumed for our calculations. The Sachs-Wolfe effect sourced by the magnetic energy density of a stochastic primordial magnetic field is presented in Sec. III. The full mode-coupling calculations are then presented for the four-point correlation of magnetic energy density. In Sec. IV we present the Sachs-Wolfe effect and four-point calculation for magnetic scalar anisotropic stress. The magnetic CMB trispectrum is then calculated for energy density and scalar anisotropic stress in Sec. $\mathrm{V}$ Additionally, in Sec. VI, the trispectrum sourced by magnetic scalar anisotropic stress is also calculated using the flat-sky approximation. Finally, in Sec. VII the Planck 2013 data release constraints on CMB non-Gaussianity [61] are used to place improved upper limits on the strength of primordial magnetic fields.

\section{PRIMORDIAL MAGNETIC FIELD}

We consider a Gaussian random stochastic magnetic field B characterized and completely specified by its power spectrum $M(k)$. We further assume that the magnetic field is nonhelical. On scales that are galactic and larger, any velocity induced by Lorentz forces is generally too small to appreciably distort the initial magnetic field [63, 64]. Therefore, the magnetic field simply redshifts away as $\mathbf{B}(\mathbf{x}, t)=\boldsymbol{b}_{0}(\mathbf{x}) / a^{2}$, where, $\boldsymbol{b}_{0}$ is the magnetic field at the present epoch (i.e. at $z=0$ or $a=1$ ). We define $\boldsymbol{b}(\boldsymbol{k})$ as the Fourier transform of the magnetic field $\boldsymbol{b}_{0}(\boldsymbol{x})$. The magnetic field power spectrum is defined as

$$
\left\langle b_{i}(\boldsymbol{k}) b_{j}^{*}(\boldsymbol{q})\right\rangle=(2 \pi)^{3} \delta(\boldsymbol{k}-\boldsymbol{q}) P_{i j}(\boldsymbol{k}) M(k)
$$

where $P_{i j}(\boldsymbol{k})=\left(\delta_{i j}-k_{i} k_{j} / k^{2}\right)$ is the projection operator ensuring $\nabla \cdot \boldsymbol{b}_{0}=0$. This gives $\left\langle\boldsymbol{b}_{0}^{2}\right\rangle=2 \int(d k / k) \Delta_{b}^{2}(k)$, where $\Delta_{b}^{2}(k)=k^{3} M(k) /\left(2 \pi^{2}\right)$ is the power per logarithmic interval in $k$-space present in the stochastic magnetic field. We also assume a power-law magnetic power spectrum, $M(k)=A k^{n}$ that is cutoff at $k=k_{c}$, where $k_{c}$ is the Alfvén-wave damping length-scale [63, 64]. We then fix the normalization $A$ by setting the variance of the magnetic field to be $B_{0}$, smoothed using a sharp $k$-space filter, over a 'galactic' scale $k_{G}=1 \mathrm{~h}$ $\mathrm{Mpc}^{-1}$. This gives, (for $n \gtrsim-3$ and for $k<k_{c}$ )

$$
\Delta_{b}^{2}(k)=\frac{k^{3} M(k)}{2 \pi^{2}}=\frac{B_{0}^{2}}{2}(n+3)\left(\frac{k}{k_{G}}\right)^{3+n} .
$$

We restrict the magnetic spectral index to values near and above -3 , i.e an inflation-generated field, as causal generation mechanisms necessarily produce much bluer magnetic power spectra [65]. Furthermore, blue spectral indices, on large scales, are strongly disfavoured by many observational constraints on primordial magnetic fields like the CMB power spectra [29-33].

\section{CMB ANISOTROPY FROM MAGNETIC ENERGY DENSITY AND FOUR-POINT CORRELATION}

The Sachs-Wolfe type of contribution to the CMB temperature anisotropy sourced by the energy density of magnetic fields [66-68], can be written as

$$
\frac{\Delta T}{T}(\boldsymbol{n})=\mathcal{R} \Omega_{B}\left(\boldsymbol{x}_{0}-\boldsymbol{n} D^{*}\right)
$$

Here, $\Omega_{B}(\boldsymbol{x})=\mathbf{B}^{2}(\boldsymbol{x}, t) /\left(8 \pi \rho_{\gamma}(t)\right)=\boldsymbol{b}_{0}^{2}(\boldsymbol{x}) /\left(8 \pi \rho_{0}\right)$, where $\rho_{\gamma}(t)$ and $\rho_{0}$ are the CMB energy densities at times $t$ and at the present epoch, respectively. Like the usual SachsWolfe effect, the $\Delta T / T$ given above is for large-angular scales. For calculating numerical values we adopt the $\mathcal{R}$ value estimated by Bonvin and Caprini (Eq. 6.12 of [68]) which is expressed according to our definitions as $\mathcal{R}=-R_{\gamma} / 15 \sim$ -0.04 , where $R_{\gamma} \sim 0.6$ is the fractional contribution of radiation energy density towards the total energy density of the relativistic component. The unit vector $\mathbf{n}$ is defined along the direction of observation from the observer at position $\boldsymbol{x}_{0}$ and $D^{*}$ is the (comoving angular diameter) distance to the surface of last scattering. We have assumed instantaneous recombination which is a good approximation for large angular scales.

The temperature fluctuations of the CMB can be expanded in terms of spherical harmonics to give $\Delta T(\boldsymbol{n}) / T=$ $\sum_{l m} a_{l m} Y_{l m}(\boldsymbol{n})$, where

$$
a_{l m}=\frac{4 \pi}{i^{l}} \int \frac{d^{3} k}{(2 \pi)^{3}} \mathcal{R} \Omega_{B}(\boldsymbol{k}) j_{l}\left(k D^{*}\right) Y_{l m}^{*}(\hat{\boldsymbol{k}}) .
$$

Note that $\Omega_{B}(\boldsymbol{k})$ is the Fourier transform of $\Omega_{B}(\boldsymbol{x})$. As $\Omega_{B}(\boldsymbol{x})$ is quadratic in $\boldsymbol{b}_{0}(\boldsymbol{x}), \Omega_{B}(\boldsymbol{k})$ is given by the convolution integral

$$
\Omega_{B}(\boldsymbol{k})=\left(1 /(2 \pi)^{3}\right) \int d^{3} s b_{i}(\boldsymbol{k}+\boldsymbol{s}) b_{i}^{*}(\boldsymbol{s}) /\left(8 \pi \rho_{0}\right) .
$$

The trispectrum $T^{m} m_{1} m_{2} m_{3} m_{4} l_{4}$, or the four-point correlation function of the $\mathrm{CMB}$ temperature anisotropy in harmonic space, in terms of the $a_{l m}$ 's is

$$
T_{l_{1} l_{2} l_{3} l_{4}}^{m_{1} m_{2} m_{3} m_{4}}=\left\langle a_{l_{1} m_{1}} a_{l_{2} m_{2}} a_{l_{3} m_{3}} a_{l_{4} m_{4}}\right\rangle .
$$




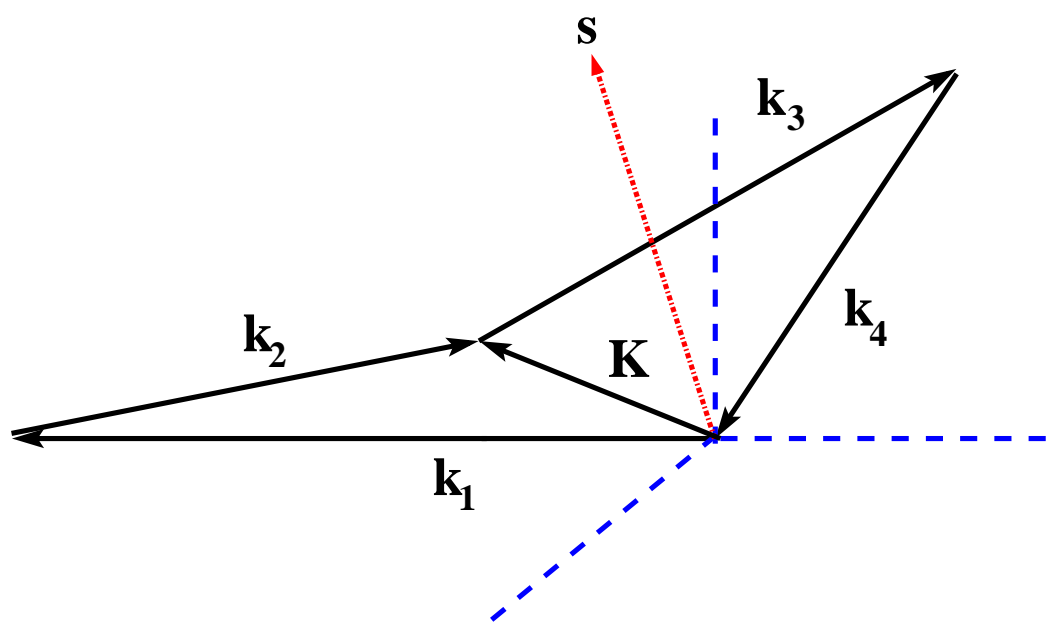

FIG. 1. The general configuration of four wavevectors $\boldsymbol{k}_{1}, \boldsymbol{k}_{2}, \boldsymbol{k}_{3}$ and $\boldsymbol{k}_{4}$ for the trispectrum with the integration mode wavevector $\boldsymbol{s}$ that appears in the mode-coupling integral.

From Eq.(4) we can express $T_{1}^{m_{1} m_{2} m_{3} m_{4}} l_{2} l_{3} l_{4}$ as

$T^{m_{1} m_{2} m_{3} m_{4} m_{2} l_{3} l_{4}}=\left(\frac{\mathcal{R}}{2 \pi^{2}}\right)^{4} \int\left[\prod_{i=1}^{4} \frac{d^{3} k_{i}}{i^{l_{i}}} j_{l_{i}}\left(k_{i} D^{*}\right) Y_{l_{i} m_{i}}^{*}\left(\hat{\boldsymbol{k}}_{i}\right)\right] \zeta_{1234}$

with

$$
\zeta_{1234}=\left\langle\Omega_{B}\left(\boldsymbol{k}_{1}\right) \Omega_{B}\left(\boldsymbol{k}_{2}\right) \Omega_{B}\left(\boldsymbol{k}_{3}\right) \Omega_{B}\left(\boldsymbol{k}_{4}\right)\right\rangle .
$$

The four-point correlation function of $\Omega_{B}(\boldsymbol{k})$ involves an eight-point correlation function of the magnetic fields. Us- ing Wick's Theorem, for Gaussian magnetic fields, we can express the magnetic eight-point correlation as a sum of 105 terms containing the magnetic two-point correlation. Neglecting 45 terms proportional to $\delta(\boldsymbol{k})$ that vanish and 12 terms proportional to $\delta\left(\boldsymbol{k}_{i}+\boldsymbol{k}_{j}\right)$ that are the unconnected part of the four-point correlation, 48 terms remain. A long calculation using the relevant projection operators gives $\zeta_{1234}=$ $\delta\left(\boldsymbol{k}_{1}+\boldsymbol{k}_{2}+\boldsymbol{k}_{3}+\boldsymbol{k}_{4}\right) \psi_{1234}$, where $\psi_{1234}$ is a mode-coupling integral over a variable $s$ and also contains angular terms.

The full expression for $\psi_{1234}$ involving angular terms in the mode-coupling integral is

$$
\begin{aligned}
\psi_{1234}=\frac{8}{\left(8 \pi \rho_{0}\right)^{4}} \int d^{3} s M(s) M\left(\left|\boldsymbol{k}_{1}+\boldsymbol{s}\right|\right) & {\left[M\left(\left|\boldsymbol{k}_{1}+\boldsymbol{k}_{3}+\boldsymbol{s}\right|\right)\left(M\left(\left|\boldsymbol{k}_{2}-\boldsymbol{s}\right|\right) \mathcal{F}_{(1)}+M\left(\left|\boldsymbol{k}_{4}-\boldsymbol{s}\right|\right) \mathcal{F}_{(2)}\right)\right.} \\
& +M\left(\left|\boldsymbol{k}_{1}+\boldsymbol{k}_{2}+\boldsymbol{s}\right|\right)\left(M\left(\left|\boldsymbol{k}_{3}-\boldsymbol{s}\right|\right) \mathcal{F}_{(3)}+M\left(\left|\boldsymbol{k}_{4}-\boldsymbol{s}\right|\right) \mathcal{F}_{(4)}\right) \\
& \left.+M\left(\left|\boldsymbol{k}_{1}+\boldsymbol{k}_{4}+\boldsymbol{s}\right|\right)\left(M\left(\left|\boldsymbol{k}_{2}-\boldsymbol{s}\right|\right) \mathcal{F}_{(5)}+M\left(\left|\boldsymbol{k}_{3}-\boldsymbol{s}\right|\right) \mathcal{F}_{(6)}\right)\right]
\end{aligned}
$$

with

$$
\begin{aligned}
& \mathcal{F}_{(1)}=-1+\left(\alpha_{1}^{2}+\alpha_{2}^{2}+\alpha_{6}^{2}+\beta_{2}^{2}+\beta_{6}^{2}+\gamma_{6}^{2}\right)-\left(\alpha_{1} \alpha_{2} \beta_{2}+\alpha_{1} \alpha_{6} \beta_{6}+\alpha_{2} \alpha_{6} \gamma_{6}+\beta_{2} \beta_{6} \gamma_{6}\right)+\alpha_{1} \alpha_{2} \beta_{6} \gamma_{6} \\
& \mathcal{F}_{(2)}=-1+\left(\alpha_{1}^{2}+\alpha_{4}^{2}+\alpha_{6}^{2}+\beta_{4}^{2}+\beta_{6}^{2}+\epsilon_{6}^{2}\right)-\left(\alpha_{1} \alpha_{4} \beta_{4}+\alpha_{1} \alpha_{6} \beta_{6}+\alpha_{4} \alpha_{6} \epsilon_{6}+\beta_{4} \beta_{6} \epsilon_{6}\right)+\alpha_{1} \alpha_{4} \beta_{6} \epsilon_{6} \\
& \mathcal{F}_{(3)}=-1+\left(\alpha_{1}^{2}+\alpha_{3}^{2}+\alpha_{5}^{2}+\beta_{3}^{2}+\beta_{5}^{2}+\delta_{5}^{2}\right)-\left(\alpha_{1} \alpha_{3} \beta_{3}+\alpha_{1} \alpha_{5} \beta_{5}+\alpha_{3} \alpha_{5} \delta_{5}+\beta_{3} \beta_{5} \delta_{5}\right)+\alpha_{1} \alpha_{3} \beta_{5} \delta_{5} \\
& \mathcal{F}_{(4)}=-1+\left(\alpha_{1}^{2}+\alpha_{4}^{2}+\alpha_{5}^{2}+\beta_{4}^{2}+\beta_{5}^{2}+\epsilon_{5}^{2}\right)-\left(\alpha_{1} \alpha_{4} \beta_{4}+\alpha_{1} \alpha_{5} \beta_{5}+\alpha_{4} \alpha_{5} \epsilon_{5}+\beta_{4} \beta_{5} \epsilon_{5}\right)+\alpha_{1} \alpha_{4} \beta_{5} \epsilon_{5} \\
& \mathcal{F}_{(5)}=-1+\left(\alpha_{1}^{2}+\alpha_{2}^{2}+\alpha_{7}^{2}+\beta_{2}^{2}+\beta_{7}^{2}+\gamma_{7}^{2}\right)-\left(\alpha_{1} \alpha_{2} \beta_{2}+\alpha_{1} \alpha_{7} \beta_{7}+\alpha_{2} \alpha_{7} \gamma_{7}+\beta_{2} \beta_{7} \gamma_{7}\right)+\alpha_{1} \alpha_{2} \beta_{7} \gamma_{7} \\
& \mathcal{F}_{(6)}=-1+\left(\alpha_{1}^{2}+\alpha_{3}^{2}+\alpha_{7}^{2}+\beta_{3}^{2}+\beta_{7}^{2}+\delta_{7}^{2}\right)-\left(\alpha_{1} \alpha_{3} \beta_{3}+\alpha_{1} \alpha_{7} \beta_{7}+\alpha_{3} \alpha_{7} \delta_{7}+\beta_{3} \beta_{7} \delta_{7}\right)+\alpha_{1} \alpha_{3} \beta_{7} \delta_{7} .
\end{aligned}
$$

The angular terms $\mathcal{F}$ contain angles defined according to

$$
\begin{aligned}
& \phi_{1}=\hat{\boldsymbol{\omega}} \cdot \widehat{\boldsymbol{k}_{1}+\boldsymbol{s}}, \phi_{2}=\hat{\boldsymbol{\omega}} \cdot \widehat{\boldsymbol{k}_{2}-\boldsymbol{s}}, \phi_{3}=\hat{\boldsymbol{\omega}} \cdot \widehat{\boldsymbol{k}_{3}-\boldsymbol{s}}, \\
& \phi_{4}=\hat{\boldsymbol{\omega}} \cdot \widehat{\boldsymbol{k}_{4}-\boldsymbol{s}}, \phi_{5}=\hat{\boldsymbol{\omega}} \cdot \widehat{\boldsymbol{k}_{1}+\boldsymbol{k}_{2}+\boldsymbol{s}}, \\
& \phi_{6}=\hat{\boldsymbol{\omega}} \cdot \widehat{\boldsymbol{k}_{1}+\boldsymbol{k}_{3}+\boldsymbol{s}}, \phi_{7}=\hat{\boldsymbol{\omega}} \cdot \widehat{\boldsymbol{k}_{1}+\boldsymbol{k}_{4}+\boldsymbol{s}},
\end{aligned}
$$

where $\widehat{\boldsymbol{k}_{1}+\boldsymbol{s}}$ is a unit vector in the direction of $\left(\boldsymbol{k}_{1}+\boldsymbol{s}\right)$ and the angle $\phi$ denotes different angles for different values of the 
unit vector $\hat{\omega}$

$$
\begin{aligned}
& \phi=\alpha \text { for } \hat{\boldsymbol{\omega}}=\hat{\boldsymbol{s}}, \quad=\beta \text { for } \hat{\boldsymbol{\omega}}=\widehat{\boldsymbol{k}_{1}+\boldsymbol{s}} \\
& \phi=\gamma \text { for } \hat{\boldsymbol{\omega}}=\widehat{\boldsymbol{k}_{2}-\boldsymbol{s}}, \quad \phi=\delta \text { for } \hat{\boldsymbol{\omega}}=\widehat{\boldsymbol{k}_{3}-\boldsymbol{s}} \\
& \phi=\epsilon \text { for } \hat{\boldsymbol{\omega}}=\widehat{\boldsymbol{k}_{4}-\boldsymbol{s}}, \phi=\kappa \text { for } \hat{\boldsymbol{\omega}}=\widehat{\boldsymbol{k}_{1}+\boldsymbol{k}_{2}+\boldsymbol{s}} \\
& \phi=\lambda \text { for } \hat{\boldsymbol{\omega}}=\widehat{\boldsymbol{k}_{1}+\boldsymbol{k}_{3}+\boldsymbol{s}} .
\end{aligned}
$$

For simplicity of calculation we evaluate the modecoupling integral $\psi_{1234}$ in two cases: (I) considering only $\boldsymbol{s}$ independent angular terms for all equal-sided configurations and (II) taking all angular terms for the collinear configuration.

\section{A. Case I - $s$-independent terms for equal-sided configurations}

Considering only $s$-independent angular terms, for a general configuration, we find $\psi_{1234}=-8 /\left(8 \pi \rho_{0}\right)^{4} \mathcal{I}$ where

$$
\begin{aligned}
& \mathcal{I}=\int d^{3} s M(s) M\left(\left|\boldsymbol{k}_{1}+\boldsymbol{s}\right|\right) \times \\
& {\left[M\left(\left|\boldsymbol{k}_{1}+\boldsymbol{k}_{3}+\boldsymbol{s}\right|\right)\left(M\left(\left|\boldsymbol{k}_{2}-\boldsymbol{s}\right|\right)+M\left(\left|\boldsymbol{k}_{4}-\boldsymbol{s}\right|\right)\right)\right.} \\
& +M\left(\left|\boldsymbol{k}_{1}+\boldsymbol{k}_{2}+\boldsymbol{s}\right|\right)\left(M\left(\left|\boldsymbol{k}_{3}-\boldsymbol{s}\right|\right)+M\left(\left|\boldsymbol{k}_{4}-\boldsymbol{s}\right|\right)\right) \\
& \left.+M\left(\left|\boldsymbol{k}_{1}+\boldsymbol{k}_{4}+\boldsymbol{s}\right|\right)\left(M\left(\left|\boldsymbol{k}_{2}-\boldsymbol{s}\right|\right)+M\left(\left|\boldsymbol{k}_{3}-\boldsymbol{s}\right|\right)\right)\right] \\
& \quad=\mathcal{I}_{(1)}+\mathcal{I}_{(2)}+\mathcal{I}_{(3)}+\mathcal{I}_{(4)}+\mathcal{I}_{(5)}+\mathcal{I}_{(6)} .
\end{aligned}
$$

We perform the mode-coupling integral employing the technique and approximations discussed in [59, 69-71], while adopting the mean (zero) value of $\hat{\boldsymbol{k}}_{1} \cdot \hat{\boldsymbol{k}}_{3}$, to find, for the first term,

$$
\mathcal{I}_{(1)} \simeq 4 \pi A^{4} k_{1}^{2 n+3} k_{2}^{n} k_{3}^{n}\left[\frac{2^{n / 2}}{n+3}-\frac{1}{4 n+3}\right] .
$$

The value of each of the $\mathcal{I}_{(j)}$ integrals for $j=1$ to 6 is the same when all the $\boldsymbol{k}_{i}$ wavevectors are of equal magnitude $\left|\boldsymbol{k}_{i}\right|=k$. We perform the $s$-independent (case I) trispectrum evaluation for such equal-sided quadrilateral configurations. Hence, $\mathcal{I}=\sum_{j=(1)}^{(6)} \mathcal{I}_{j}=6 \mathcal{I}_{(1)}$, and we obtain

$$
\begin{aligned}
& \zeta_{1234}=\delta\left(\boldsymbol{k}_{1}+\boldsymbol{k}_{2}+\boldsymbol{k}_{3}+\boldsymbol{k}_{4}\right) \times \\
& \left.\frac{-8(24 \pi) A^{4} k_{1}^{2 n+3} k_{2}^{n} k_{3}^{n}}{\left(8 \pi \rho_{0}\right)^{4}}\left[\frac{\left(2^{n / 2}\right)(4 n+3)-(n+3)}{(4 n+3)(n+3)}\right] 15\right)
\end{aligned}
$$

\section{B. Case II - Equal-Sided Collinear Configuration}

We calculate the full mode-coupling integral $\psi_{1234}$ (Eq. 9[10) (over all angular terms for each $\mathcal{F}$ expression) for the case of the equal-sided collinear configuration. All the four wavevectors are of equal magnitude with configuration $\boldsymbol{k}_{1}=$ $\boldsymbol{k}_{2}=-\boldsymbol{k}_{3}=-\boldsymbol{k}_{4}$. We find that the 28 independent angles defined by Equations 1112 reduce to just 6 independent angles $\alpha_{1}, \alpha_{2}, \alpha_{5}, \beta_{2}, \beta_{5}$ and $\gamma_{5}$. The angular expressions $\mathcal{F}$ also reduce in size from a total of 72 to 19 angular terms:

$$
\psi_{1234}^{\text {coll }}=\frac{8}{\left(8 \pi \rho_{0}\right)^{4}} \mathcal{I}^{\text {coll }}
$$

where

$$
\begin{aligned}
& \mathcal{I}^{\text {coll }}=2 \int d^{3} s M(s) M(|\boldsymbol{k}+\boldsymbol{s}|) \times \\
& \quad\left[M(s) M(|\boldsymbol{k}-\boldsymbol{s}|)\left(\alpha_{1}^{2}+\alpha_{2}^{2}+\beta_{2}^{2}-2 \alpha_{1} \alpha_{2} \beta_{2}+\alpha_{1}^{2} \alpha_{2}^{2}\right)\right. \\
& +M(s) M(|\boldsymbol{k}+\boldsymbol{s}|)\left(1+\alpha_{4}^{2}\right) \\
& +M(|2 \boldsymbol{k}+\boldsymbol{s}|) M(|\boldsymbol{k}+\boldsymbol{s}|)\left(\alpha_{1}^{2}+\alpha_{5}^{2}+\beta_{5}^{2}-\alpha_{1} \alpha_{5} \beta_{5}\right. \\
& \left.\left.\quad+\frac{1}{2}\left\{\delta_{5}^{2}+\epsilon_{5}^{2}+\left(\beta_{5}+\alpha_{1} \alpha_{5}-\alpha_{1}^{2} \beta_{5}\right)\left(\delta_{5}+\epsilon_{5}\right)\right\}\right)\right] .
\end{aligned}
$$

Using the same technique of evaluating the mode-coupling integrals as used earlier in Case I, we calculate the integrals for each of the 19 angular terms that sum together to give

$$
\mathcal{I}^{\text {coll }} \simeq 4 \pi A^{4} k_{1}^{2 n+3} k_{2}^{n} k_{3}^{n}\left[\frac{8}{3} \frac{2^{n / 2}}{n+3}-\frac{12}{4 n+3}\right] .
$$

The four-point correlation of magnetic energy density for the collinear configuration is

$$
\begin{gathered}
\zeta_{1234}=\delta\left(\boldsymbol{k}_{1}+\boldsymbol{k}_{2}+\boldsymbol{k}_{3}+\boldsymbol{k}_{4}\right) \times \\
\frac{8(4 \pi) A^{4} k_{1}^{2 n+3} k_{2}^{n} k_{3}^{n}}{\left(8 \pi \rho_{0}\right)^{4}}\left[\frac{\frac{8}{3}\left(2^{n / 2}\right)(4 n+3)-(12)(n+3)}{(4 n+3)(n+3)}\right](.19)
\end{gathered}
$$

\section{CMB ANISOTROPY FROM MAGNETIC SCALAR ANISOTROPIC STRESS AND FOUR-POINT CORRELATION}

The scalar anisotropic stress that is associated with a primordial magnetic field, in addition to its energy density, will also act as a separate source for CMB fluctuations - the passive mode [68, 72]. As we saw in our previous work [59], the magnetic scalar anisotropic stress generates $\sim 10^{6}$ times larger contribution to the $\mathrm{CMB}$ bispectrum compared to magnetic energy density. With this motivation in mind and employing the magnetic $\mathrm{CMB}$ trispectrum technique developed above, we carry out a longer calculation for the scalar anisotropic stress trispectrum.

On large angular scales, the magnetic contribution to the temperature anisotropy is again via the magnetic Sachs-Wolfe effect

$$
\frac{\Delta T}{T}(\boldsymbol{n})=\frac{1}{3} \Phi\left(\boldsymbol{x}_{0}-\boldsymbol{n} D^{*}\right)=\frac{1}{5} \zeta\left(\boldsymbol{x}_{0}-\boldsymbol{n} D^{*}\right)
$$

in the matter dominated era. We use the expression for the curvature perturbation due to the passive mode scalar anisotropic stress [72]

$$
\zeta \simeq-\frac{1}{3} R_{\gamma} \Pi_{B} \ln \left(\frac{\tau_{\nu}}{\tau_{B}}\right)
$$


to obtain temperature anisotropy, sourced by magnetic scalar anisotropic stress $\Pi_{B}$

$$
\frac{\Delta T}{T}(\boldsymbol{n})=\mathcal{R}_{p} \Pi_{B}\left(\boldsymbol{x}_{0}-\boldsymbol{n} D^{*}\right),
$$

where $\mathcal{R}_{p}=\mathcal{R} \ln \left(\tau_{\nu} / \tau_{B}\right)=\left[-R_{\gamma} / 15\right] \ln \left(T_{B} / T_{\nu}\right)$ and $\tau_{B}$ as well as $\tau_{\nu}$ and $T_{B}$ as well as $T_{\nu}$ are the conformal time and temperatures at the epochs of magnetic field generation and neutrino decoupling, respectively. None of the details of the magnetic scalar anisotropic stress calculation were included in our letter [60] and they are presented below.

The CMB temperature fluctuations can be expanded in terms of spherical harmonics to give $\Delta T(\boldsymbol{n}) / T=$ $\sum_{l m} a_{l m} Y_{l m}(\boldsymbol{n})$, where

$$
a_{l m}=\frac{4 \pi}{i^{l}} \int \frac{d^{3} k}{(2 \pi)^{3}} \mathcal{R}_{p} \Pi_{B}(\boldsymbol{k}) j_{l}\left(k D^{*}\right) Y_{l m}^{*}(\hat{\boldsymbol{k}}) .
$$

Here, $\Pi_{B}(\boldsymbol{k})$ is the Fourier transform of $\Pi_{B}(\boldsymbol{x})$ and we recall the operator that projects out the scalar anisotropic stress from the full magnetic stress $\Pi_{B}^{i j}(\boldsymbol{k})$

$$
\Pi_{B}(\boldsymbol{k})=\frac{1}{2}\left(\delta_{i j}-3 \hat{\boldsymbol{k}}_{i} \hat{\boldsymbol{k}}_{j}\right) \Pi_{B}^{i j}(\boldsymbol{k})
$$

Since $\Pi_{B}(\boldsymbol{x})$ is quadratic in $\boldsymbol{b}_{0}(\boldsymbol{x})$, we have a convolution of magnetic fields

$$
\Pi_{B}(\boldsymbol{k})=\frac{1}{2}\left(\delta_{i j}-3 \hat{\boldsymbol{k}}_{i} \hat{\boldsymbol{k}}_{j}\right) \frac{1}{4 \pi p_{\gamma}} \int \frac{d^{3} s}{(2 \pi)^{3}} \boldsymbol{b}_{i}^{*}(\boldsymbol{s}) \boldsymbol{b}_{j}(\boldsymbol{k}+\boldsymbol{s})
$$

The trispectrum is $T_{l_{1}}^{m_{1} m_{2} m_{3} m_{3}} l_{4} l_{4}=\left\langle a_{l_{1} m_{1}} a_{l_{2} m_{2}} a_{l_{3} m_{3}} a_{l_{4} m_{4}}\right\rangle$, is then given by

$T_{l_{1} l_{2} l_{3} l_{4}}^{m_{1} m_{2} m_{3} m_{4}}=\left(\frac{\mathcal{R}_{p}}{2 \pi^{2}}\right)^{4} \int\left[\prod_{i=1}^{4} \frac{d^{3} k_{i}}{i^{l_{i}}} j_{l_{i}}\left(k_{i} D^{*}\right) Y_{l_{i} m_{i}}^{*}\left(\hat{\boldsymbol{k}}_{i}\right)\right]\left[\zeta_{1234}\right]_{\Pi}$

with $\left[\zeta_{1234}\right]_{\Pi}$ defined as

$$
\left[\zeta_{1234}\right]_{\Pi}=\left\langle\Pi_{B}\left(\boldsymbol{k}_{1}\right) \Pi_{B}\left(\boldsymbol{k}_{2}\right) \Pi_{B}\left(\boldsymbol{k}_{3}\right) \Pi_{B}\left(\boldsymbol{k}_{4}\right)\right\rangle .
$$

The four-point correlation function of $\Pi_{B}(\boldsymbol{k})$, like that of $\Omega_{B}(\boldsymbol{k})$, also involves an eight-point correlation function of the fields. In similar fashion, using Wick's Theorem, for Gaussian magnetic fields, we express the magnetic eight-point correlation as a sum of 105 terms involving the magnetic two-point correlation function. Then 45 terms proportional to $\delta(\boldsymbol{k})$ vanish and we neglect the 12 terms proportional to $\delta\left(\boldsymbol{k}_{i}+\boldsymbol{k}_{j}\right)$ that represent the unconnected part of the fourpoint correlation, to leave 48 terms. A long calculation involving the relevant projection operators in these terms gives $\left[\zeta_{1234}\right]_{\Pi}=\delta\left(\boldsymbol{k}_{1}+\boldsymbol{k}_{2}+\boldsymbol{k}_{3}+\boldsymbol{k}_{4}\right)\left[\psi_{1234}\right]_{\Pi}$, where $\left[\psi_{1234}\right]_{\Pi}$ is a mode-coupling integral over a variable $s$ and also involves angular terms. The key difference between the $\Omega_{B}$ and the $\Pi_{B}$ four-point correlations is the number and type of operators acting on the magnetic field eight-point correlation. In the case of energy density $\Omega_{B}$, the operator $\delta_{a b} \delta_{c d} \delta_{e f} \delta_{g h}$ acted on

$$
\left\langle\boldsymbol{b}_{a}(-s) \boldsymbol{b}_{b}\left(\boldsymbol{k}_{1}+\boldsymbol{s}\right) \boldsymbol{b}_{c}(-\boldsymbol{r}) \boldsymbol{b}_{d}\left(\boldsymbol{k}_{2}+\boldsymbol{r}\right) \boldsymbol{b}_{e}(-\boldsymbol{t}) \boldsymbol{b}_{f}\left(\boldsymbol{k}_{3}+\boldsymbol{t}\right) \boldsymbol{b}_{g}(-\boldsymbol{w}) \boldsymbol{b}_{h}\left(\boldsymbol{k}_{4}+\boldsymbol{w}\right)\right\rangle
$$

However, in the case of scalar anisotropic stress $\Pi_{B}$, there are 16 operator terms

$$
\begin{aligned}
\left(\delta_{a b}-3 \hat{\boldsymbol{k}}_{1_{a}} \hat{\boldsymbol{k}}_{1_{b}}\right)\left(\delta_{c d}-3 \hat{\boldsymbol{k}}_{2_{c}} \hat{\boldsymbol{k}}_{2_{d}}\right)\left(\delta_{e f}-3 \hat{\boldsymbol{k}}_{3_{e}} \hat{\boldsymbol{k}}_{3_{f}}\right)\left(\delta_{g h}-3 \hat{\boldsymbol{k}}_{4_{g}} \hat{\boldsymbol{k}}_{4_{h}}\right) \\
=\delta_{a b} \delta_{c d} \delta_{e f} \delta_{g h}-3\left[\delta_{a b} \delta_{c d} \delta_{e f} \hat{\boldsymbol{k}}_{4_{g}} \hat{\boldsymbol{k}}_{4_{h}}+\delta_{a b} \delta_{c d} \hat{\boldsymbol{k}}_{3_{e}} \hat{\boldsymbol{k}}_{3_{f}} \delta_{g h}+\delta_{a b} \hat{\boldsymbol{k}}_{2_{c}} \hat{\boldsymbol{k}}_{2_{d}} \delta_{e f} \delta_{g h}+\hat{\boldsymbol{k}}_{1_{a}} \hat{\boldsymbol{k}}_{1_{b}} \delta_{c d} \delta_{e f} \delta_{g h}\right] \\
\quad+9\left[\delta_{a b} \delta_{c d} \hat{\boldsymbol{k}}_{3_{e}} \hat{\boldsymbol{k}}_{3_{f}} \hat{\boldsymbol{k}}_{4_{g}} \hat{\boldsymbol{k}}_{4_{h}}+\delta_{a b} \hat{\boldsymbol{k}}_{2_{c}} \hat{\boldsymbol{k}}_{2_{d}} \delta_{e f} \hat{\boldsymbol{k}}_{4_{g}} \hat{\boldsymbol{k}}_{4_{h}}+\delta_{a b} \hat{\boldsymbol{k}}_{2_{c}} \hat{\boldsymbol{k}}_{2_{d}} \hat{\boldsymbol{k}}_{3_{e}} \hat{\boldsymbol{k}}_{3_{f}} \delta_{g h}+\hat{\boldsymbol{k}}_{1_{a}} \hat{\boldsymbol{k}}_{1_{b}} \delta_{c d} \delta_{e f} \hat{\boldsymbol{k}}_{4_{g}} \hat{\boldsymbol{k}}_{4_{h}}\right. \\
\left.\quad+\hat{\boldsymbol{k}}_{1_{a}} \hat{\boldsymbol{k}}_{1_{b}} \delta_{c d} \hat{\boldsymbol{k}}_{3_{e}} \hat{\boldsymbol{k}}_{3_{f}} \delta_{g h}+\hat{\boldsymbol{k}}_{1_{a}} \hat{\boldsymbol{k}}_{1_{b}} \hat{\boldsymbol{k}}_{2_{c}} \hat{\boldsymbol{k}}_{2_{d}} \delta_{e f} \delta_{g h}\right] \\
\quad-27\left[\delta_{a b} \hat{\boldsymbol{k}}_{2_{c}} \hat{\boldsymbol{k}}_{2_{d}} \hat{\boldsymbol{k}}_{3_{e}} \hat{\boldsymbol{k}}_{3_{f}} \hat{\boldsymbol{k}}_{4_{g}} \hat{\boldsymbol{k}}_{4_{h}}+\hat{\boldsymbol{k}}_{1_{a}} \hat{\boldsymbol{k}}_{1_{b}} \delta_{c d} \hat{\boldsymbol{k}}_{3_{e}} \hat{\boldsymbol{k}}_{3_{f}} \hat{\boldsymbol{k}}_{4_{g}} \hat{\boldsymbol{k}}_{4_{h}}+\hat{\boldsymbol{k}}_{1_{a}} \hat{\boldsymbol{k}}_{1_{b}} \hat{\boldsymbol{k}}_{2_{c}} \hat{\boldsymbol{k}}_{2_{d}} \delta_{e f} \hat{\boldsymbol{k}}_{4_{g}} \hat{\boldsymbol{k}}_{4_{h}}+\hat{\boldsymbol{k}}_{1_{a}} \hat{\boldsymbol{k}}_{1_{b}} \hat{\boldsymbol{k}}_{2_{c}} \hat{\boldsymbol{k}}_{2_{d}} \hat{\boldsymbol{k}}_{3_{e}} \hat{\boldsymbol{k}}_{3_{f}} \delta_{g h}\right] \\
\quad+81 \hat{\boldsymbol{k}}_{1_{a}} \hat{\boldsymbol{k}}_{1_{b}} \hat{\boldsymbol{k}}_{2_{c}} \hat{\boldsymbol{k}}_{2_{d}} \hat{\boldsymbol{k}}_{3_{e}} \hat{\boldsymbol{k}}_{3_{f}} \hat{\boldsymbol{k}}_{4_{g}} \hat{\boldsymbol{k}}_{4_{h}} \\
=1
\end{aligned}
$$

Each operator term $X$ from 1 to 16 generates its own separate angular term expression $\mathcal{F} \frac{\mathrm{x}}{(I)}$. When summed over all $X$ this yields the angular term expression $\mathcal{F}_{(I)}$, where $I$ takes values 1 to 6 in the six term mode-coupling integral $\left[\psi_{1234}\right]_{\Pi}$. As operator 1 is identical to the operator for the $\Omega_{B}$ four-point correlation, the angular terms $\mathcal{F}$ for it are just given by Equation 10 . We give below the expressions for $\left[\psi_{1234}\right]_{\Pi}$ and the angular terms $\mathcal{F}$ generated by operators 2 and 16 , suppressing the $\Pi$ subscript. The complete expression for the full set of over 1500 angular terms generated by all sixteen operators 1 through 16 
TABLE I. Angle definitions for scalar anisotropic stress $\Pi_{B}$ angular terms, with $i=1$ to 4 .

\begin{tabular}{|c|c|c|c|c|c|c|c|}
\hline $\bar{\alpha}_{i}$ & $\bar{\beta}_{i}$ & $\bar{\gamma}_{i}$ & $\bar{\delta}_{i}$ & $\bar{\epsilon}_{i}$ & $\bar{\kappa}_{i}$ & $\bar{\lambda}_{i}$ & $\bar{\chi}_{i}$ \\
\hline$\hat{\boldsymbol{k}}_{i} \cdot \hat{\boldsymbol{s}}$ & $\hat{\boldsymbol{k}}_{i} \cdot \widehat{\boldsymbol{k}_{1}+\boldsymbol{s}}$ & $\hat{\boldsymbol{k}}_{i} \cdot \widehat{\boldsymbol{k}_{2}-\boldsymbol{s}}$ & $\hat{\boldsymbol{k}}_{i} \cdot \widehat{\boldsymbol{k}_{3}-\boldsymbol{s}}$ & $\hat{\boldsymbol{k}}_{i} \cdot \widehat{\boldsymbol{k}_{4}-\boldsymbol{s}}$ & $\hat{\boldsymbol{k}}_{i} \cdot \overline{\boldsymbol{k}_{1}+\boldsymbol{k}_{2}+\boldsymbol{s}}$ & $\hat{\boldsymbol{k}}_{i} \cdot \overline{\boldsymbol{k}_{1}+\boldsymbol{k}_{3}+\boldsymbol{s}}$ & $\hat{\boldsymbol{k}}_{i} \cdot \widehat{\boldsymbol{k}_{1}+\boldsymbol{k}_{4}+\boldsymbol{s}}$ \\
\hline
\end{tabular}

is placed in Appendix A. The mode-coupling integral for scalar anisotropic stress is,

$$
\begin{aligned}
{\left[\psi_{1234}\right]_{\Pi}=\frac{8}{\left(8 \pi p_{0}\right)^{4}} \int d^{3} s M(s) M\left(\left|\boldsymbol{k}_{1}+\boldsymbol{s}\right|\right) } & {\left[M\left(\left|\boldsymbol{k}_{1}+\boldsymbol{k}_{3}+\boldsymbol{s}\right|\right)\left(M\left(\left|\boldsymbol{k}_{2}-\boldsymbol{s}\right|\right) \mathcal{F}_{(1)}+M\left(\left|\boldsymbol{k}_{4}-\boldsymbol{s}\right|\right) \mathcal{F}_{(2)}\right)\right.} \\
& +M\left(\left|\boldsymbol{k}_{1}+\boldsymbol{k}_{2}+\boldsymbol{s}\right|\right)\left(M\left(\left|\boldsymbol{k}_{3}-\boldsymbol{s}\right|\right) \mathcal{F}_{(3)}+M\left(\left|\boldsymbol{k}_{4}-\boldsymbol{s}\right|\right) \mathcal{F}_{(4)}\right) \\
& \left.+M\left(\left|\boldsymbol{k}_{1}+\boldsymbol{k}_{4}+\boldsymbol{s}\right|\right)\left(M\left(\left|\boldsymbol{k}_{2}-\boldsymbol{s}\right|\right) \mathcal{F}_{(5)}+M\left(\left|\boldsymbol{k}_{3}-\boldsymbol{s}\right|\right) \mathcal{F}_{(6)}\right)\right]
\end{aligned}
$$

where $p_{0}=\rho_{0} / 3$ and $\rho_{0}$ is the present-day energy density in radiation. The angular expressions $\mathcal{F}$ now involve 32 new angles (with overbars) defined below, in addition to the 28 previously defined angles (without overbars) that appear in the $\Omega_{B}$ expression - Equations (11/12). The new angles defined in Table \arise from dot products of the four $\hat{k}$ wavevectors with the vector $\hat{s}$ or with those combinations of $s$ and the four $\hat{\boldsymbol{k}}$ wavevectors that appear in the equation for $\psi_{1234}$.

The angular terms for operator 2 are

$$
\begin{aligned}
& \mathcal{F} \frac{2}{(1)}=1-\bar{\beta}_{4}^{2}-\bar{\alpha}_{4}^{2}+\alpha_{1} \bar{\alpha}_{4} \bar{\beta}_{4}-\bar{\lambda}_{4}\left[\bar{\lambda}_{4}-\bar{\beta}_{4} \beta_{6}-\bar{\alpha}_{4} \alpha_{6}+\alpha_{1} \bar{\alpha}_{4} \beta_{6}\right]-\bar{\gamma}_{4}\left[\bar{\gamma}_{4}-\bar{\beta}_{4} \beta_{2}-\bar{\alpha}_{4} \alpha_{2}+\alpha_{1} \bar{\beta}_{4} \alpha_{2}\right] \\
& +\bar{\gamma}_{4} \bar{\lambda}_{4}\left[\gamma_{6}-\beta_{2} \beta_{6}-\alpha_{2} \alpha_{6}+\alpha_{1} \alpha_{2} \beta_{6}\right] \\
& \mathcal{F}_{(2)}^{2}=1-\bar{\beta}_{4}^{2}-\bar{\lambda}_{4}^{2}+\beta_{6} \bar{\lambda}_{4} \bar{\beta}_{4}-\bar{\epsilon}_{4}\left[\bar{\epsilon}_{4}-\bar{\beta}_{4} \beta_{6}-\bar{\lambda}_{4} \epsilon_{6}+\beta_{6} \bar{\beta}_{4} \epsilon_{6}\right]-\bar{\alpha}_{4}\left[\bar{\alpha}_{4}-\bar{\beta}_{4} \alpha_{1}-\bar{\lambda}_{4} \alpha_{6}+\beta_{6} \bar{\lambda}_{4} \alpha_{1}\right] \\
& +\bar{\alpha}_{4} \bar{\epsilon}_{4}\left[\alpha_{4}-\alpha_{1} \beta_{4}-\alpha_{6} \epsilon_{6}+\beta_{6} \alpha_{1} \epsilon_{6}\right] \\
& \mathcal{F} \stackrel{2}{(3)}=1-\bar{\beta}_{4}^{2}-\bar{\alpha}_{4}^{2}+\alpha_{1} \bar{\alpha}_{4} \bar{\beta}_{4}-\bar{\kappa}_{4}\left[\bar{\kappa}_{4}-\bar{\beta}_{4} \beta_{5}-\bar{\alpha}_{4} \alpha_{5}+\alpha_{1} \bar{\alpha}_{4} \beta_{5}\right]-\bar{\delta}_{4}\left[\bar{\delta}_{4}-\bar{\beta}_{4} \beta_{3}-\bar{\alpha}_{4} \alpha_{3}+\alpha_{1} \bar{\beta}_{4} \alpha_{3}\right] \\
& +\bar{\delta}_{4} \bar{\kappa}_{4}\left[\delta_{5}-\beta_{3} \beta_{5}-\alpha_{3} \alpha_{5}+\alpha_{1} \alpha_{3} \beta_{5}\right] \\
& \mathcal{F} \frac{2}{(4)}=1-\bar{\beta}_{4}^{2}-\bar{\kappa}_{4}^{2}+\beta_{5} \bar{\kappa}_{4} \bar{\beta}_{4}-\bar{\epsilon}_{4}\left[\bar{\epsilon}_{4}-\bar{\kappa}_{4} \epsilon_{5}-\bar{\beta}_{4} \beta_{4}+\beta_{5} \bar{\beta}_{4} \epsilon_{5}\right]-\bar{\alpha}_{4}\left[\bar{\alpha}_{4}-\bar{\kappa}_{4} \alpha_{5}-\bar{\beta}_{4} \alpha_{1}+\beta_{5} \bar{\kappa}_{4} \alpha_{1}\right] \\
& +\bar{\alpha}_{4} \bar{\epsilon}_{4}\left[\alpha_{4}-\alpha_{5} \epsilon_{5}-\alpha_{1} \beta_{4}+\beta_{5} \alpha_{1} \epsilon_{5}\right] \\
& \mathcal{F} \frac{2}{(5)}=1-\bar{\gamma}_{4}^{2}-\bar{\alpha}_{4}^{2}+\alpha_{2} \bar{\alpha}_{4} \bar{\gamma}_{4}-\bar{\chi}_{4}\left[\bar{\chi}_{4}-\bar{\gamma}_{4} \gamma_{7}-\bar{\alpha}_{4} \alpha_{7}+\alpha_{2} \bar{\alpha}_{4} \gamma_{7}\right]-\bar{\beta}_{4}\left[\bar{\beta}_{4}-\bar{\gamma}_{4} \beta_{2}-\bar{\alpha}_{4} \alpha_{1}+\alpha_{2} \bar{\gamma}_{4} \alpha_{1}\right] \\
& +\bar{\beta}_{4} \bar{\chi}_{4}\left[\beta_{7}-\beta_{2} \gamma_{7}-\alpha_{1} \alpha_{7}+\alpha_{2} \gamma_{7} \alpha_{1}\right] \\
& \mathcal{F} \frac{2}{(6)}=1-\bar{\alpha}_{4}^{2}-\bar{\delta}_{4}^{2}+\alpha_{3} \bar{\alpha}_{4} \bar{\delta}_{4}-\bar{\chi}_{4}\left[\bar{\chi}_{4}-\bar{\alpha}_{4} \alpha_{7}-\bar{\delta}_{4} \delta_{7}+\alpha_{3} \bar{\alpha}_{4} \delta_{7}\right]-\bar{\beta}_{4}\left[\bar{\beta}_{4}-\bar{\alpha}_{4} \alpha_{1}-\bar{\delta}_{4} \beta_{3}+\alpha_{3} \bar{\delta}_{4} \alpha_{1}\right] \\
& +\bar{\beta}_{4} \bar{\chi}_{4}\left[\beta_{7}-\alpha_{1} \alpha_{7}-\beta_{3} \delta_{7}+\alpha_{3} \alpha_{1} \delta_{7}\right]
\end{aligned}
$$

and angular terms for operator 16 are

$$
\begin{aligned}
& \mathcal{F} \frac{16}{(1)}=\left(\theta_{12}-\bar{\alpha}_{1} \bar{\alpha}_{2}\right)\left(\theta_{13}-\bar{\beta}_{1} \bar{\beta}_{3}\right)\left(\theta_{24}-\bar{\gamma}_{2} \bar{\gamma}_{4}\right)\left(\theta_{34}-\bar{\lambda}_{3} \bar{\lambda}_{4}\right) \\
& \mathcal{F} \frac{16}{(2)}=\left(\theta_{14}-\bar{\alpha}_{1} \bar{\alpha}_{4}\right)\left(\theta_{13}-\bar{\beta}_{1} \bar{\beta}_{3}\right)\left(\theta_{24}-\bar{\epsilon}_{2} \bar{\epsilon}_{4}\right)\left(\theta_{23}-\bar{\lambda}_{2} \bar{\lambda}_{3}\right) \\
& \mathcal{F} \frac{\sqrt{(3)}}{(3)}=\left(\theta_{13}-\bar{\alpha}_{1} \bar{\alpha}_{3}\right)\left(\theta_{12}-\bar{\beta}_{1} \bar{\beta}_{2}\right)\left(\theta_{34}-\bar{\delta}_{3} \bar{\delta}_{4}\right)\left(\theta_{24}-\bar{\kappa}_{2} \bar{\kappa}_{4}\right) \\
& \mathcal{F} \frac{16}{(4)}=\left(\theta_{14}-\bar{\alpha}_{1} \bar{\alpha}_{4}\right)\left(\theta_{12}-\bar{\beta}_{1} \bar{\beta}_{2}\right)\left(\theta_{34}-\bar{\epsilon}_{3} \bar{\epsilon}_{4}\right)\left(\theta_{23}-\bar{\kappa}_{2} \bar{\kappa}_{3}\right) \\
& \mathcal{F} \frac{16}{(5)}=\left(\theta_{12}-\bar{\alpha}_{1} \bar{\alpha}_{2}\right)\left(\theta_{14}-\bar{\beta}_{1} \bar{\beta}_{4}\right)\left(\theta_{23}-\bar{\gamma}_{2} \bar{\gamma}_{3}\right)\left(\theta_{34}-\bar{\chi}_{3} \bar{\chi}_{4}\right) \\
& \mathcal{F}-\frac{16}{(6)}=\left(\theta_{13}-\bar{\alpha}_{1} \bar{\alpha}_{3}\right)\left(\theta_{14}-\bar{\beta}_{1} \bar{\beta}_{4}\right)\left(\theta_{23}-\bar{\delta}_{2} \bar{\delta}_{3}\right)\left(\theta_{24}-\bar{\chi}_{2} \bar{\chi}_{4}\right) \text {. }
\end{aligned}
$$

In addition to the angles defined in Table $\llbracket$ angular terms

like $\theta_{a b}=\hat{\boldsymbol{k}}_{a} \cdot \hat{\boldsymbol{k}}_{b}$ that are constant for a given $\left(\boldsymbol{k}_{1}, \boldsymbol{k}_{2}, \boldsymbol{k}_{3}, \boldsymbol{k}_{4}\right)$ 


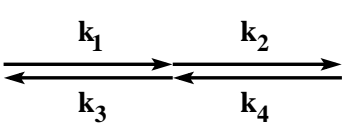

(i) collinear

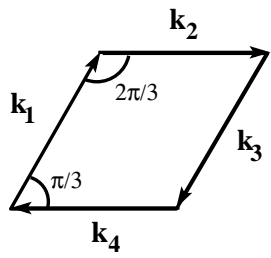

(iii) rhombus

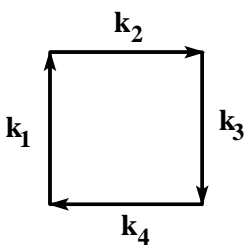

(ii) square

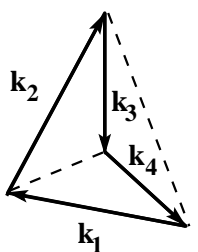

(iv) tetrahedral
FIG. 2. The four specific configurations (i) collinear, (ii) square, (iii) rhombus and (iv) tetrahedral, with each wavevector of equal magnitude $k$, used to evaluate the magnetic scalar anisotropic trispectrum.

configuration also appear. In total, as pointed out above, over 1500 angular terms are present in all the $\mathcal{F}$ expressions for $\left[\psi_{1234}\right]_{\Pi}$, many more than the 72 terms for $\left[\psi_{1234}\right]_{\Omega}$. To arrive at a representative estimate for $\left[\psi_{1234}\right]_{\Pi}$, we consider only the $s$-independent angular terms and restrict ourselves to equalsided trispectrum configurations i.e. all $\left|\boldsymbol{k}_{i}\right| \simeq k$. The $s$ independent terms are

$$
\begin{aligned}
& \mathcal{F}_{\Pi}^{\text {s-indep }}=6\left[-13+9\left(\theta_{12}^{2}+\theta_{13}^{2}+\theta_{14}^{2}+\theta_{23}^{2}+\theta_{24}^{2}+\theta_{34}^{2}\right)\right. \\
& -27\left(\theta_{12} \theta_{13} \theta_{23}+\theta_{12} \theta_{14} \theta_{24}+\theta_{13} \theta_{14} \theta_{34}+\theta_{23} \theta_{24} \theta_{34}\right) \\
& \left.+27\left(\theta_{12} \theta_{13} \theta_{24} \theta_{34}+\theta_{12} \theta_{14} \theta_{23} \theta_{34}+\theta_{13} \theta_{14} \theta_{23} \theta_{24}\right)\right] .
\end{aligned}
$$

We evaluate $\mathcal{F}_{\Pi}^{\text {s-indep }}$ for specific equal-sided trispectrum configurations: collinear, square, rhombus and tetrahedral. Table III lists the values of $\mathcal{F}_{\Pi}^{\text {s-indep }}$ for the specific configurations $\left(\boldsymbol{k}_{1}, \boldsymbol{k}_{2}, \boldsymbol{k}_{3}, \boldsymbol{k}_{4}\right)$, showing that the greatest contribution to $\left[\psi_{1234}\right]_{\Pi}$ and therefore to the scalar anisotropic stress trispectrum arises from the collinear configuration. The values for $\mathcal{F}_{\Pi}^{\text {s-indep }}$ range from $\approx-2$ to 14 . We adopt a value of 10 as a typical value for the sum of all $s$-independent terms and denote it by $\xi$. We get a mode-coupling integral with an integrand that matches the $\left[\psi_{1234}\right]_{\Omega}$ for the $\Omega_{B} s$-independent equal-sided configuration case I Equation (13)

$$
\left[\psi_{1234}\right]_{\Pi}=\frac{8 \xi}{\left(8 \pi p_{0}\right)^{4}} \mathcal{I}=\frac{8\left(3^{4}\right) \xi}{\left(8 \pi \rho_{0}\right)^{4}} \mathcal{I}
$$

where

$$
\begin{aligned}
& \mathcal{I}=\int d^{3} s M(s) M\left(\left|\boldsymbol{k}_{1}+\boldsymbol{s}\right|\right) \times \\
& {\left[M\left(\left|\boldsymbol{k}_{1}+\boldsymbol{k}_{3}+\boldsymbol{s}\right|\right)\left(M\left(\left|\boldsymbol{k}_{2}-\boldsymbol{s}\right|\right)+M\left(\left|\boldsymbol{k}_{4}-\boldsymbol{s}\right|\right)\right)\right.} \\
& +M\left(\left|\boldsymbol{k}_{1}+\boldsymbol{k}_{2}+\boldsymbol{s}\right|\right)\left(M\left(\left|\boldsymbol{k}_{3}-\boldsymbol{s}\right|\right)+M\left(\left|\boldsymbol{k}_{4}-\boldsymbol{s}\right|\right)\right) \\
& \left.+M\left(\left|\boldsymbol{k}_{1}+\boldsymbol{k}_{4}+\boldsymbol{s}\right|\right)\left(M\left(\left|\boldsymbol{k}_{2}-\boldsymbol{s}\right|\right)+M\left(\left|\boldsymbol{k}_{3}-\boldsymbol{s}\right|\right)\right)\right](35)
\end{aligned}
$$

TABLE II. The value of the $s$-independent terms $\mathcal{F}_{\Pi}^{\text {s-indep }}$ in four different equal-sided configurations $\left(\boldsymbol{k}_{1}, \boldsymbol{k}_{2}, \boldsymbol{k}_{3}, \boldsymbol{k}_{4}\right)$ with $k_{1} \sim k_{2} \sim k_{3} \sim k_{4}$ for evaluating the magnetic scalar anisotropic stress trispectrum.

\begin{tabular}{ccc}
\hline \hline Configuration & $\left(\theta_{12}, \theta_{13}, \theta_{14}, \theta_{23}, \theta_{24}, \theta_{34}\right)$ & $\mathcal{F}_{\Pi}^{\text {s-indep }}$ \\
\hline \hline collinear & $(1,-1,-1,-1,-1,1)$ & 14 \\
\hline square & $(0,-1,0,0,-1,0)$ & 5 \\
\hline rhombus & $\left(\frac{1}{2},-1,-\frac{1}{2},-\frac{1}{2},-1, \frac{1}{2}\right)$ & 2.1875 \\
\hline tetrahedral & $\left(-\frac{1}{2}, 0,-\frac{1}{2},-\frac{1}{2}, 0,-\frac{1}{2}\right)$ & -2.3125 \\
\hline \hline
\end{tabular}

The integral $\mathcal{I}$ is evaluated as earlier to yield the four-point correlation of the magnetic scalar anisotropic stress to be

$$
\left[\zeta_{1234}\right]_{\Pi}=\delta\left(\boldsymbol{k}_{1}+\boldsymbol{k}_{2}+\boldsymbol{k}_{3}+\boldsymbol{k}_{4}\right) \times
$$

$3^{4} \xi \frac{8(24 \pi) A^{4} k_{1}^{2 n+3} k_{2}^{n} k_{3}^{n}}{\left(8 \pi \rho_{0}\right)^{4}}\left[\frac{\left(2^{n / 2}\right)(4 n+3)-(n+3)}{(4 n+3)(n+3)}\right](36)$

or simply expressed, in relation to the four-point correlation of energy density,

$$
\left[\zeta_{1234}\right]_{\Pi}=3^{4} \xi\left[-\zeta_{1234}\right]_{\Omega} .
$$

\section{MAGNETIC CMB TRISPECTRUM}

Having calculated the four-point correlations, in Fourier space, of energy density $\left[\zeta_{1234}\right]_{\Omega}$ and scalar anisotropic stress $\left[\zeta_{1234}\right]_{\Pi}$, we can now calculate the CMB trispectrum sourced by each.

\section{A. CMB Trispectrum from Magnetic Energy Density}

For the trispectrum sourced by magnetic energy density $\Omega_{B}$, we insert Eq. (15) into Eq. (7) for the trispectrum and following the approach of [73, 74], we decompose our delta function as $\delta\left(\boldsymbol{k}_{1}+\boldsymbol{k}_{2}+\boldsymbol{k}_{3}+\boldsymbol{k}_{4}\right)=\int d^{3} K \delta\left(\boldsymbol{k}_{1}+\boldsymbol{k}_{2}+\right.$ $\boldsymbol{K}) \delta\left(\boldsymbol{k}_{3}+\boldsymbol{k}_{4}-\boldsymbol{K}\right)$. We can then write the trispectrum as

$$
\begin{aligned}
& T_{l_{1} l_{2} l_{3} l_{4}}^{m_{m_{3}} m_{3} m_{3} m_{4}}=(4 \pi)^{4}(-1)^{\sum_{i} l_{i}} \mathcal{R}^{4} \int \frac{d^{3} k_{1} d^{3} k_{2} d^{3} k_{3} d^{3} k_{4}}{(2 \pi)^{12}} \\
& \times j_{l_{1}}\left(k_{1} D^{*}\right) j_{l_{2}}\left(k_{2} D^{*}\right) j_{l_{3}}\left(k_{3} D^{*}\right) j_{l_{4}}\left(k_{4} D^{*}\right) \\
& \times Y_{l_{1} m_{1}}^{*}\left(\hat{\boldsymbol{k}}_{1}\right) Y_{l_{2} m_{2}}^{*}\left(\hat{\boldsymbol{k}}_{2}\right) Y_{l_{3} m_{3}}^{*}\left(\hat{\boldsymbol{k}}_{3}\right) Y_{l_{4} m_{4}}^{*}\left(\hat{\boldsymbol{k}}_{4}\right) \\
& \times\left[\frac{-(192 \pi) A^{4}}{\left(8 \pi \rho_{0}\right)^{4}} k_{1}^{2 n+3} k_{2}^{n} k_{3}^{n}\left\{\frac{\left(2^{n / 2}\right)(4 n+3)-(n+3)}{(4 n+3)(n+3)}\right\}\right] \\
& \times \int d^{3} K \delta\left(\boldsymbol{k}_{1}+\boldsymbol{k}_{2}+\boldsymbol{K}\right) \delta\left(\boldsymbol{k}_{3}+\boldsymbol{k}_{4}-\boldsymbol{K}\right) .
\end{aligned}
$$


Using the integral form of the delta functions

$$
\begin{gathered}
\int d^{3} K \delta\left(\boldsymbol{k}_{1}+\boldsymbol{k}_{2}+\boldsymbol{K}\right) \delta\left(\boldsymbol{k}_{3}+\boldsymbol{k}_{4}-\boldsymbol{K}\right)= \\
\int \frac{d^{3} K}{(2 \pi)^{6}} \int d^{3} r_{1}^{2} \int d^{3} r_{2}^{2} e^{\left(\boldsymbol{k}_{1}+\boldsymbol{k}_{2}+\boldsymbol{K}\right) \cdot \boldsymbol{r}_{1}} e^{\left(\boldsymbol{k}_{3}+\boldsymbol{k}_{4}-\boldsymbol{K}\right) \cdot \boldsymbol{r}_{2}},
\end{gathered}
$$

and the spherical wave expansion

$$
e^{i \boldsymbol{k}_{j} \cdot \boldsymbol{r}}=4 \pi \sum_{l^{\prime}=0}^{\infty} i^{l^{\prime}} j_{l^{\prime}}\left(k_{j} r\right) \sum_{m^{\prime}=-l^{\prime}}^{+l^{\prime}} Y_{l^{\prime} m^{\prime}}^{*}\left(\hat{\boldsymbol{k}}_{j}\right) Y_{l^{\prime} m^{\prime}}(\hat{\boldsymbol{r}})
$$

we perform the integrals over the angular parts of $\left(\boldsymbol{k}_{1}, \boldsymbol{k}_{2}, \boldsymbol{k}_{3}, \boldsymbol{k}_{4}, \boldsymbol{K}\right)$, with algebra similar to [49, 59, 75, 76], to give

$$
\begin{aligned}
& T_{l_{1} l_{2} l_{3} l_{4} m_{2} m_{4}}^{m_{2} m_{2}}\left[(-768) \frac{\mathcal{R}^{4}}{\pi^{7}}\right]\left(\frac{A}{\left(8 \pi \rho_{0}\right)}\right)^{4} \\
& \times\left\{\frac{\left(2^{n / 2}\right)(4 n+3)-(n+3)}{(4 n+3)(n+3)}\right\} \\
& \times \int d r_{1} r_{1}^{2} \int d r_{2} r_{2}^{2} \int d k_{1} k_{1}^{2} k_{1}^{2 n+3} j_{l_{1}}\left(k_{1} D^{*}\right) j_{l_{1}}\left(k_{1} r_{1}\right) \\
& \times \int d k_{2} k_{2}^{2} k_{2}^{n} j_{l_{2}}\left(k_{2} D^{*}\right) j_{l_{2}}\left(k_{2} r_{1}\right) \int d k_{3} k_{3}^{2} k_{3}^{n} j_{l_{3}}\left(k_{3} D^{*}\right) j_{l_{3}}\left(k_{3} r_{2}\right) \\
& \times \int d k_{4} k_{4}^{2} j_{l_{4}}\left(k_{4} D^{*}\right) j_{l_{4}}\left(k_{4} r_{2}\right) \times \sum_{L M}(-1)^{L-M} \\
& \times \int d K K^{2} j_{L}\left(K r_{1}\right) j_{L}\left(-K r_{2}\right) \\
& \times \int d \Omega_{\hat{\boldsymbol{r}}_{1}} Y_{l_{1} m_{1}}\left(\hat{\boldsymbol{r}}_{1}\right) Y_{l_{2} m_{2}}\left(\hat{\boldsymbol{r}}_{1}\right) Y_{L M}\left(\hat{\boldsymbol{r}}_{1}\right) \\
& \times \int d \Omega_{\hat{\boldsymbol{r}}_{2}} Y_{l_{3} m_{3}}\left(\hat{\boldsymbol{r}}_{2}\right) Y_{l_{4} m_{4}}\left(\hat{\boldsymbol{r}}_{2}\right) Y_{L-M}\left(\hat{\boldsymbol{r}}_{2}\right) .
\end{aligned}
$$

Here the $K$-integral gives $\delta\left(r_{1}-r_{2}\right)\left(\pi / 2 r_{1}^{2}\right)$ using the spherical Bessel function closure relation. This delta function enables us to perform the $r_{2}$-integral trivially, then $r_{1}$ replaces $r_{2}$ in the arguments of $j_{l_{3}}$ and $j_{l_{4}}$. The angular $\hat{\boldsymbol{r}}_{1}$ and $\hat{\boldsymbol{r}}_{2^{-}}$ integrals may be expressed as (e.g. Eq. 5.9.1 (5) of [77])

$$
\begin{aligned}
& \int d \Omega_{\hat{\boldsymbol{r}}_{1}} Y_{l_{1} m_{1}}\left(\hat{\boldsymbol{r}}_{1}\right) Y_{l_{2} m_{2}}\left(\hat{\boldsymbol{r}}_{1}\right) Y_{L M}\left(\hat{\boldsymbol{r}}_{1}\right)= \\
& \sqrt{\frac{\left(2 l_{1}+1\right)\left(2 l_{2}+1\right)(2 L+1)}{4 \pi}}\left(\begin{array}{ccc}
l_{1} & l_{2} & L \\
0 & 0 & 0
\end{array}\right)\left(\begin{array}{ccc}
l_{1} & l_{2} & L \\
m_{1} & m_{2} & M
\end{array}\right) \\
& \equiv h_{l_{1} L l_{2}}\left(\begin{array}{ccc}
l_{1} & l_{2} & L \\
m_{1} & m_{2} & M
\end{array}\right)
\end{aligned}
$$

where we have defined $h_{l_{1} L l_{2}}$ above, in the same convention as $[73,74]$. We use the relation

$$
\left(A / 8 \pi \rho_{0}\right)^{4}=(2 / 3)^{4}\left(\pi / k_{G}\right)^{8}\left((n+3) / k_{G}^{n+1}\right)^{4} V_{A}{ }^{8},
$$

where the Alfvén velocity $V_{A}$, in the radiation dominated era, is defined as [63, 64],

$$
V_{A}=B_{0} /\left(16 \pi \rho_{0} / 3\right)^{1 / 2} \approx 3.8 \times 10^{-4} B_{-9}
$$

with $B_{-9} \equiv\left(B_{0} / 10^{-9}\right.$ Gauss $)$. From the definition of the rotationally invariant angle-averaged trispectrum [78]

$$
\begin{aligned}
T_{l_{1} l_{2} l_{3} l_{4}}^{m_{1} m_{2} m_{3} m_{4}}= & \sum_{L M}(-1)^{-M}\left(\begin{array}{ccc}
l_{1} & l_{2} & L \\
m_{1} & m_{2} & -M
\end{array}\right) \\
& \times\left(\begin{array}{ccc}
l_{3} & l_{4} & L \\
m_{3} & m_{4} & M
\end{array}\right) T_{l_{3} l_{4}}^{l_{1} l_{2}}(L),
\end{aligned}
$$

we separate out the reduced trispectrum $T_{l_{3} l_{1}}^{l_{1} l_{2}}(L)$ (referred to as the angular averaged trispectrum in [78]), from the full trispectrum. We again use the spherical Bessel function closure relation to perform the $k_{4}$-integral that yields $\delta\left(r_{1}-D^{*}\right)\left(\pi / 2 r_{1}^{2}\right)$. This facilitates the $r_{1}$-integral that results in $r_{1} \rightarrow D^{*}$ in the arguments of $j_{l_{1}}, j_{l_{2}}$ and $j_{l_{3}}$. The $k_{1}, k_{2}$ and $k_{3}$-integrals containing a product of a power-law and $j_{l}^{2}$ can be evaluated in terms of Gamma functions (e.g. Eq. 6.574 .2 of [79]). For a scale-invariant magnetic index $n \rightarrow-3$, we get

$$
\begin{aligned}
{\left[T_{l_{3} l_{4}}^{l_{1} l_{2}}(L)\right]_{\Omega} \simeq } & -5.8 \times 10^{-29}\left(\frac{n+3}{0.2}\right)^{3}\left(\frac{B-9}{3}\right)^{8} \\
& \times \frac{h_{l_{1} L l_{2}} h_{l_{3} L l_{4}}}{l_{1}\left(l_{1}+1\right) l_{2}\left(l_{2}+1\right) l_{3}\left(l_{3}+1\right)}
\end{aligned}
$$

This equation gives us the amplitude of the magnetic CMB trispectrum sourced by the energy density $\Omega_{B}$ of a primordial magnetic field, where we have used $\mathcal{R} \sim-0.04$ [68]. A factor of $1 /\left(D^{*} k_{G}\right)^{4(n+3)}$ also appears here and it approaches unity for the case $n \rightarrow-3$ (a scale-invariant magnetic field index). When we evaluate the magnetic trispectrum for a near scaleinvariant index $n=-2.8$, this factor has a value $\sim 1 / 1500$. It then turns out that this factor is almost entirely canceled by the simultaneous increase in the value of the $k$-integrals when evaluated for $n=-2.8$ rather than $n=-3$.

For the case II - collinear configuration case, proceeding from Eq. [19] in exactly the same way as case I, we find that the amplitude of the collinear configuration trispectrum is

$$
\begin{aligned}
{\left[T_{l_{3} l_{4}}^{l_{1} l_{2}}(L)\right]_{\Omega} \simeq } & 3.9 \times 10^{-29}\left(\frac{n+3}{0.2}\right)^{3}\left(\frac{B-9}{3}\right)^{8} \\
& \times \frac{h_{l_{1} L l_{2}} h_{l_{3} L l_{4}}}{l_{1}\left(l_{1}+1\right) l_{2}\left(l_{2}+1\right) l_{3}\left(l_{3}+1\right)}
\end{aligned}
$$

which is similar in magnitude to the case I trispectrum, but of positive sign.

\section{B. CMB Trispectrum from Magnetic Scalar Anisotropic Stress}

The scalar anisotropic stress trispectrum $\left[T_{l_{3} l_{4}}^{l_{1} l_{2}}(L)\right]_{\Pi}$ can be calculated in an analogous manner to the calculation presented above for case I $s$-independent $\left[T_{l_{3} l_{4}}^{l_{1} l_{2}}(L)\right]_{\Omega}$. Using 
Equations (26) and (36) we obtain

$$
\begin{aligned}
{\left[T_{l_{3} l_{4}}^{l_{1} l_{2}}(L)\right]_{\Pi} \simeq } & \left(3 \frac{\mathcal{R}_{p}}{\mathcal{R}}\right)^{4} \xi\left[-T_{l_{3} l_{4}}^{l_{1} l_{2}}(L)\right]_{\Omega} \\
\simeq & 1.1 \times 10^{-19}\left(\frac{\xi}{10}\right)\left(\frac{n+3}{0.2}\right)^{3}\left(\frac{B-9}{3}\right)^{8} \\
& \times \frac{h_{l_{1} L l_{2} h_{l_{3} L l_{4}}}}{l_{1}\left(l_{1}+1\right) l_{2}\left(l_{2}+1\right) l_{3}\left(l_{3}+1\right)} .
\end{aligned}
$$

We see that the amplitude of the trispectrum sourced by $\Pi_{B}$ for equal-sided quadrilateral configurations is approximately $10^{10}$ times larger than that sourced by $\Omega_{B}$. Here, we have used $T_{B} \simeq 10^{14} \mathrm{GeV}$ (corresponding to the reheating temperature) and $T_{\nu} \simeq 10^{-3} \mathrm{GeV}$.

\section{FLAT-SKY CALCULATION OF SCALAR ANISOTROPIC STRESS CMB TRISPECTRUM}

We now consider a flat-sky analysis of the trispectrum. The flat-sky limit allows us to avoid the approximate treatment of the angular terms involving $\hat{\boldsymbol{k}}_{i}$ while performing the $\hat{\boldsymbol{k}}$ angular integrals that led to Equation (41). Therefore, to get a more accurate estimate of the $s$-independent anisotropic stress trispectrum, we now adopt the flat-sky limit for the CMB temperature anisotropy and recompute the trispectrum.

In the flat-sky limit [80-82], the CMB temperature fluctuations on the sky are expanded in terms of plane waves using a Fourier basis rather than a spherical harmonic basis,

$$
\begin{aligned}
\frac{\Delta T}{T}(\boldsymbol{n}) & =\int \frac{d^{2} l}{(2 \pi)^{2}} a_{\ell} e^{i \boldsymbol{\ell} \cdot \boldsymbol{n}}, \\
a_{\ell} & =\int d^{2} n \frac{\Delta T}{T}(\boldsymbol{n}) e^{-i \boldsymbol{\ell} \cdot \boldsymbol{n}} .
\end{aligned}
$$

In the flat-sky co-ordinates, $\ell=\left(\ell_{x}, \ell_{y}\right)$ is a two-dimensional vector on the plane of the sky and $n_{z}$ is a constant equal to unity at linear order. In order to check the validity of our flatsky technique, we first computed the magnetic energy density bispectrum. We find a value for the flat-sky bispectrum of order $\approx 10^{-23}$, which agrees well with the original full-sky result [49]. This encourages us to proceed to the flat-sky limit calculation of the scalar anisotropic stress trispectrum.

The magnetic Sachs-Wolfe effect for scalar anisotropic stress is given by

$$
\begin{aligned}
\frac{\Delta T}{T}(\boldsymbol{n}) & =\mathcal{R}_{p} \Pi_{B}\left(\boldsymbol{x}_{0}-\boldsymbol{n} D^{*}\right) \\
& =\int \frac{d^{3} k}{(2 \pi)^{3}} \mathcal{R}_{p} \Pi_{B}(\boldsymbol{k}) e^{i \boldsymbol{k} \cdot\left(\boldsymbol{x}_{0}-\boldsymbol{n}_{D^{*}}\right)} \\
& =\mathcal{R}_{p} \int \frac{d^{3} k}{(2 \pi)^{3}} \Pi_{B}(\boldsymbol{k}) e^{-i(\boldsymbol{k} \cdot \boldsymbol{n}) D^{*}} .
\end{aligned}
$$

where in the last line we set the observer's position $\boldsymbol{x}_{0}$ to the origin.

The flat-sky limit is accurate for $\ell \gtrsim 40$ [80-82] whereas the Sachs-Wolfe contribution is appreciable for $\ell \lesssim 100$ (but dominant only till $\ell \lesssim 50$ ) [83]. Therefore, there exists an appreciable range of overlap $40 \lesssim \ell \lesssim 100$ in harmonic space, where we can treat the Sachs-Wolfe contribution to the CMB temperature anisotropy in the flat-sky limit.

In the flat-sky limit, $n_{z}$ is constant and is unity to linear order hence $\boldsymbol{n} \cdot \boldsymbol{k} \rightarrow \boldsymbol{m} \cdot \boldsymbol{k}_{\perp}+k_{z}$ which gives

$$
\begin{aligned}
a_{\ell} & =\int d^{2} n\left(\frac{\Delta T}{T}(\boldsymbol{n})\right)_{\text {flat sky }} e^{-i \boldsymbol{\ell} \cdot \boldsymbol{n}} \\
& =\mathcal{R}_{p} \int \frac{d^{3} k}{(2 \pi)^{3}} \Pi_{B}(\boldsymbol{k}) e^{-i k_{z} D^{*}} \int d^{2} m e^{-i \boldsymbol{m} \cdot\left(\boldsymbol{\ell}+\boldsymbol{k}_{\perp} D^{*}\right)} .
\end{aligned}
$$

The $m$-integral gives a delta function for $\boldsymbol{k}_{\perp}$

$$
\begin{gathered}
\int d^{2} m e^{-i \boldsymbol{m} \cdot\left(\boldsymbol{\ell}+\boldsymbol{k}_{\perp} D^{*}\right)}=(2 \pi)^{2} \delta^{(2)}\left(\boldsymbol{\ell}+\boldsymbol{k}_{\perp} D^{*}\right) \\
=\left(\frac{2 \pi}{D^{*}}\right)^{2} \delta^{(2)}\left(\frac{\boldsymbol{\ell}}{D^{*}}+\boldsymbol{k}_{\perp}\right)
\end{gathered}
$$

to yield

$$
a_{\ell}=\frac{\mathcal{R}_{p}}{\left(D^{*}\right)^{2}} \int_{\infty}^{\infty} \frac{d k_{z}}{2 \pi} \Pi_{B}\left(\boldsymbol{k}_{\perp}=\frac{-\ell}{D^{*}}, k_{z}\right) e^{-i k_{z} D^{*}} .
$$

This flat-sky $a_{\ell}$ for magnetic scalar anisotropic stress can then be used to calculate the corresponding trispectrum in the flat-sky limit

$$
\left\langle a_{\ell_{1}} a_{\ell_{2}} a_{\ell_{3}} a_{\ell_{4}}\right\rangle=\left(\frac{\mathcal{R}_{p}}{2 \pi}\right)^{4}\left[\prod_{i=1}^{4} \int_{\infty}^{\infty} d k_{i_{z}} \frac{e^{-i k_{i_{z}} D_{i}^{*}}}{\left(D_{i}^{*}\right)^{2}}\right] \zeta_{1234}^{\mathrm{fs}}
$$

where $\zeta_{1234}^{\mathrm{fs}}$ is the four-point correlation of magnetic scalar anisotropic stress in the flat-sky limit

$$
\zeta_{1234}^{\mathrm{fs}}=\left\langle\left[\prod_{i=1}^{4} \Pi_{B}\left(\boldsymbol{k}_{i_{\perp}}=\frac{-\ell_{i}}{D_{i}^{*}}, k_{i_{z}}\right)\right]\right\rangle .
$$

As before in the full-sky for $\zeta_{1234}$ (Eqs. 27, 36), a four-point correlation of $\Pi_{B}$ produces delta functions times a mode coupling integral $\psi$.

$$
\begin{aligned}
\zeta_{1234}^{\mathrm{fs}}= & \delta\left(k_{1_{z}}+k_{2_{z}}+k_{3_{z}}+k_{4_{z}}\right) \\
& \times \delta^{(2)}\left(\frac{\ell_{1}}{D_{1}^{*}}+\frac{\ell_{2}}{D_{2}^{*}}+\frac{\ell_{3}}{D_{3}^{*}}+\frac{\ell_{4}}{D_{4}^{*}}\right)\left[\psi_{1234}\right]_{\Pi}^{\mathrm{fs}}
\end{aligned}
$$

If we take the $D_{i}^{*}$ 's to be similar, we find

$$
\begin{aligned}
\zeta_{1234}^{\mathrm{fs}}= & \delta\left(k_{1_{z}}+k_{2_{z}}+k_{3 z}+k_{4_{z}}\right) \\
& \times\left(D^{*}\right)^{2} \delta^{(2)}\left(\boldsymbol{\ell}_{1}+\boldsymbol{\ell}_{2}+\boldsymbol{\ell}_{3}+\boldsymbol{\ell}_{4}\right)\left[\psi_{1234}\right]_{\Pi}^{\mathrm{fs}}
\end{aligned}
$$

Here the mode-coupling integral $\psi$ is

$$
\left[\psi_{1234}\right]_{\Pi}^{\mathrm{fs}}=\frac{8 \mathcal{F}_{\Pi}^{\mathrm{s} \text {-indep }}}{\left(8 \pi p_{0}\right)^{4}} \mathcal{I}
$$

where the integral $\mathcal{I}$ is the same as the one given by Eq. 35 and the $s$-independent angular terms for $\Pi_{B}$ are denoted by $\mathcal{F}_{\Pi}^{\text {s-indep }}$ given by Eq. (33). In the flat-sky approach we perform the mode-coupling integral for general values of $\hat{\boldsymbol{k}}_{i} \cdot \hat{\boldsymbol{k}}_{j}$ 
TABLE III. The value of $\sigma$ [the product of the integral $\mathcal{I}$ Eq. 35] and $k_{i_{z}}$ integrals Eq. 55] for the three different trispectrum configurations (shown in Fig. 3) considered for the flat-sky magnetic scalar anisotropic stress trispectrum (Eq. 62.

\begin{tabular}{cccc}
\hline \hline Configuration & $\left(q_{12}, q_{13}, q_{14}, q_{23}, q_{24}, q_{34}\right)$ & $\left(\hat{\ell}_{1} \cdot \hat{\ell}_{2}, \hat{\ell}_{1} \cdot \hat{\ell}_{3}, \hat{\ell}_{1} \cdot \hat{\ell}_{4}, \hat{\ell}_{2} \cdot \hat{\ell}_{3}, \hat{\ell}_{2} \cdot \hat{\ell}_{4}, \hat{\ell}_{3} \cdot \hat{\ell}_{4}\right)$ & $\sigma$ \\
\hline \hline \multirow{2}{*}{ kite } & $(\sqrt{3}, \sqrt{3}, 1,1,1 / \sqrt{3}, 1 / \sqrt{3})$ & $(0,-\sqrt{3} / 2,-1 / 2,1 / 2,-\sqrt{3} / 2,0)$ & -15.2 \\
\hline \multirow{2}{*}{ trapezium } & $(2,2 / 3,2,1 / 3,1,3)$ & $(1 / 2,-1,1 / 2,-1 / 2,-1 / 2,-1 / 2)$ & -84.6 \\
\hline \multirow{2}{*}{ scalene } & $(1 / 3,2 / 3,0.4406,2,1.322,0.6609)$ & $(0,-\sqrt{3} / 2,0.1317,-1 / 2,-0.9912,0.3815)$ & -14.2 \\
\hline \hline
\end{tabular}



(i) kite

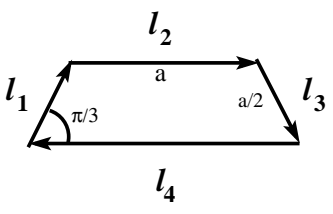

(ii) trapezium

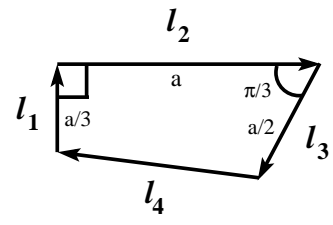

(iii) scalene

FIG. 3. The three specific $\ell$ wavevector configurations (i) kite, (ii) trapezium (both cyclic quadrilaterals) and (iii) scalene (an irregular convex quadrilateral) used to evaluate the flat-sky magnetic scalar anisotropic trispectrum. Trispectrum configuration shapes (i) and (ii) are also discussed in [84, 85]

and later evaluate the trispectrum for particular configurations that are not necessarily equal-sided. The first term (out of six terms) of integral $\mathcal{I}$ is

$\mathcal{I}_{(1)}^{\mathrm{fs}} \simeq 4 \pi A^{4} k_{1}^{2 n+3} k_{2}^{n}\left[\frac{\left(k_{1}^{2}+2 k_{1} k_{3} \theta_{13}+k_{3}^{2}\right)^{n / 2}}{n+3}-\frac{k_{3}^{n}}{4 n+3}\right]$

Whereas, in the full-sky $\Pi_{B}$ calculation we chose a representative value $\xi$ for $\mathcal{F}_{\Pi}^{\text {s-indep }}$, we now integrate over all 14 terms of $\mathcal{F}_{\Pi}^{\text {s-indep }}$ in the $k_{i_{z}}$ integrals.

For each of the 6 terms of $\mathcal{I}$, the delta function of $k_{i_{z}}$ is used to perform that particular $k_{i_{z}}$ integral (one out of four)

\footnotetext{
${ }^{1}$ For some highly symmetrical configurations which have two $\ell$ vectors exactly anti-parallel and of equal magnitude, the $k_{z}$ integral becomes singular in the flat-sky limit. However, this is due to the exact $\boldsymbol{k}_{i_{\perp}}=\frac{-\boldsymbol{\ell}_{i}}{D_{i}^{*}}$ map which is enforced in this limit. If this were relaxed then we expect
}

for which the variable $k_{i}$ that does not appear in the arguments of the magnetic spectrum $M$. This introduces substitutions in the angular structure $\mathcal{F}_{\Pi}^{\text {s-indep }}$. Then the remaining three $k_{i_{z}}$ integrals are performed numerically and evaluated for several types of configurations. We use the relation for the flat-sky trispectrum (connected part) [73, 74]

$$
\left\langle a_{\ell_{1}} a_{\ell_{2}} a_{\ell_{3}} a_{\ell_{4}}\right\rangle=(2 \pi)^{2} \delta^{(2)}\left(\ell_{1}+\ell_{2}+\ell_{3}+\ell_{4}\right) T_{\left(\ell_{3}, \ell_{4}\right)}^{\left(\ell_{1}, \ell_{2}\right)}(L)
$$

to get $\left[T_{\left(\ell_{3}, \ell_{4}\right)}^{\left(\ell_{1}, \ell_{2}\right)}(L)\right]_{\Pi}$ from the four-point correlation of $a_{\ell}$. The product of the mode coupling integral $\mathcal{I}$ and the three $k_{i_{z}}$ integrals is denoted by $\sigma$. Table (III) shows different values of $\sigma$ for different $\ell$-space configurations with parameters $q_{a b}=l_{a} / l_{b}$ (ratio of different sides) and $\hat{\ell}_{i} \cdot \hat{\ell}_{j}$ (cosine of the angle between sides). We note that all the configurations thus evaluated in the flat-sky approach (for all s-independent terms) give a negative $\sigma$ that lead to a negative value of the trispectrum 1

$$
\begin{aligned}
{\left[T_{\left(\ell_{3}, \ell_{4}\right)}^{\left(\ell_{1}, \ell_{2}\right)}(L)\right]_{\Pi} \simeq } & 3.94 \times 10^{-19}\left(\frac{\sigma}{10}\right)\left(\frac{n+3}{0.2}\right)^{3}\left(\frac{B_{-9}}{3}\right)^{8} \\
\times & \frac{1}{l_{1}^{2} l_{2}^{2} l_{3}^{2}} .
\end{aligned}
$$

We see that the flat-sky evaluation of the scalar anisotropic stress trispectrum with $s$-independent terms results in trispectra that are negative and roughly an order of magnitude larger in absolute magnitude than the corresponding full-sky trispectrum with $s$-independent terms (with $\xi \approx 10$ ). The flat-sky and full-sky trispectra are related by

$$
T_{\left(\ell_{3}, \ell_{4}\right)}^{\left(\ell_{1}, \ell_{2}\right)}(L) h_{l_{1} L l_{2}} h_{l_{3} L l_{4}} \approx T_{l_{3} l_{4}}^{l_{1} l_{2}}(L)
$$

This allows us to compare the flat-sky trispectrum directly to the full-sky trispectrum form given in Eq. 48

this mathematical pathology to be just an integrable singularity. The measure of such configurations in $d^{3} k$ is expected to go to zero faster than the reciprocal of the integrand. 


\section{PRIMORDIAL MAGNETIC FIELD CONSTRAINTS}

We can now compare our magnetic trispectra with the Sachs-Wolfe contribution to the standard CMB trispectrum sourced by non-linear terms in the inflationary perturbations calculated by Okamoto \& Hu [73] and Kogo \& Komatsu [74] (also see [47]).

$$
\begin{aligned}
T_{l_{3} l_{4}}^{l_{1} l_{2}}(L) \approx & 9 C_{l_{2}}^{S W} C_{l_{4}}^{S W}\left[(25 / 9) \tau_{N L} C_{L}^{S W}\right. \\
& \left.+6 g_{N L}\left(C_{l_{1}}^{S W}+C_{l_{3}}^{S W}\right)\right] h_{l_{1} L l_{2}} h_{l_{3} L l_{4}}(64)
\end{aligned}
$$

We neglect the $g_{N L}$ term that places far weaker constraints on the trispectrum compared to the $\tau_{N L}$ term considering the the current limits on $g_{N L}$ from WMAP [86] and current limits on $\tau_{N L}$ from Planck [61]. The CMB angular power spectrum $C_{l}^{S W}$ in the Sachs-Wolfe approximation for a scale-invariant primordial power spectrum for $\Phi$ is

$$
C_{l}^{S W}=\frac{2}{9 \pi} \int k^{2} d k P_{\Phi}(k) j_{l}^{2}\left(k r_{*}\right)=\frac{A_{\Phi}}{l(l+1)},
$$

where $A_{\Phi}$ is the amplitude of scalar potential perturbations. This gives

$$
\begin{aligned}
T_{l_{3} l_{4}}^{l_{1} l_{2}}(L) \approx 25 C_{l_{2}}^{S W} C_{l_{4}}^{S W} C_{L}^{S W} \tau_{N L} h_{l_{1} L l_{2}} h_{l_{3} L l_{4}} \\
\approx 25 A_{\Phi}^{3} \tau_{N L} \frac{h_{l_{1} L l_{2}} h_{l_{3} L l_{4}}}{l_{2}\left(l_{2}+1\right) l_{4}\left(l_{4}+1\right) L(L+1)} \\
\approx 25 A_{\Phi}^{3} \tau_{N L} \frac{h_{l_{1} L l_{2}} h_{l_{3} L l_{4}}}{l_{1}\left(l_{1}+1\right) l_{2}\left(l_{2}+1\right) l_{3}\left(l_{3}+1\right)} \\
\quad \times \frac{l_{1}\left(l_{1}+1\right) l_{3}\left(l_{3}+1\right)}{l_{4}\left(l_{4}+1\right) L(L+1)} \\
\approx 25 A_{\Phi}^{3} \tau_{N L} \frac{h_{l_{1} L l_{2}} h_{l_{3} L l_{4}}}{l_{1}\left(l_{1}+1\right) l_{2}\left(l_{2}+1\right) l_{3}\left(l_{3}+1\right)} q,
\end{aligned}
$$

where we also define a factor $q=$ $\left[l_{1}\left(l_{1}+1\right) l_{3}\left(l_{3}+1\right)\right] /\left[l_{4}\left(l_{4}+1\right) L(L+1)\right]$ which is of order unity for many configurations. To calculate the value of $A_{\Phi}$ we begin with the most recent Planck 2013 data release value for the amplitude of scalar curvature perturbations on [29] $A_{s}=2.2 \times 10^{-9}$ at a pivot scale $k_{0}=0.05 \mathrm{Mpc}^{-1}$. For the purpose of the Sachs-Wolfe contribution we then calculate the scalar amplitude at the larger scale of $k_{0}=0.002 \mathrm{Mpc}^{-1}$ using the Planck 2013 value for the scalar spectral index $n_{s}=0.96$. After converting from curvature to potential we get $A_{\Phi}=6.96 \times 10^{-10}$. Hence, we find the amplitude for the Sachs-Wolfe contribution to the standard CMB trispectrum sourced by inflationary perturbations to be

$T_{l_{3} l_{4}}^{l_{1} l_{2}}(L) \approx 8.4 \times 10^{-27} \tau_{N L} \frac{h_{l_{1} L l_{2}} h_{l_{3} L l_{4}}}{l_{1}\left(l_{1}+1\right) l_{2}\left(l_{2}+1\right) l_{3}\left(l_{3}+1\right)} q$.

Equation (67) is of the same form as Eq. (46) and Eq. (48) for the magnetic field-induced trispectra, facilitating direct comparison of trispectra values.

\section{A. Limits from Magnetic Energy Density - Case I}

We can put upper limits on the primordial magnetic field by comparing the magnetic energy density trispectrum Eq. (46) with the inflationary trispectrum Eq. 67), although stronger constraints follow from magnetic anisotropic stress. We take the two-sigma upper limit value on $\tau_{N L}$ reported in the Planck 2013 data release: $\tau_{N L}<2,800$ [61] and use it also as a lower limit for possible negative values of $\tau_{N L}$ i.e. $\left|\tau_{N L}\right|<2,800$. This is tighter than the $\tau_{N L}>-6,000$ negative-sided limit from WMAP5 data [86] that we employed in [60]. Magnetic field limits are obtained by taking the one-eighth power of the appropriate ratio of trispectra, which gives $B_{0} \lesssim 19 \mathrm{nG}$ at a scale of $k_{G}=1 \mathrm{~h} \mathrm{Mpc}^{-1}$ for a magnetic spectral index of $n=-2.8$. This trispectrum limit is almost a factor of 2 stronger than the bispectrum upper limit $B_{0} \lesssim 35 \mathrm{nG}$ found for magnetic energy density [49] for the same scale and magnetic index.

We note that if we update the value of $\mathcal{R}$ used in the earlier bispectrum calculation [49] to the currently adopted value of $\mathcal{R}$ [68] then the magnetic energy density bispectrum yields a tighter upper limit of $B_{0} \lesssim 30 \mathrm{nG}$. The trispectrum constraint we calculated above, $B_{0} \lesssim 19 \mathrm{nG}$, seems significantly stronger than the bisectrum constraint (by a factor of 1.6). However, since the energy density bispectrum calculation [49] was performed, the $f_{N L}^{l o c}$ two-sigma upper limit has tightened from $\approx 100$ (WMAP5) [87] to 74 (WMAP7) [88] to 14.3 (Planck 2013) 61]. Recalculation of the magnetic field constraint from the magnetic energy density bispectrum, now using $f_{N L}^{l o c}<14.3$, yields $B_{0} \lesssim 22 \mathrm{nG}$. We see that the corresponding magnetic energy density trispectrum limit (19 $\mathrm{nG})$ found in this work is, nevertheless, slightly stronger than the updated bispectrum limit.

\section{B. Limits from Magnetic Energy Density - Collinear Configuration}

We have also calculated the magnetic energy density trispectrum considering all the angular terms that appear for the collinear configuration (case II). Comparing the collinear configuration energy density trispectrum Eq. 477 to the inflationary trispectrum Eq. 67) leads to upper limits on the primordial magnetic field of $B_{0} \lesssim 20 \mathrm{nG}$, having employed the positive-sided limit $\tau_{N L}<2,800$ [61]. This $B_{0}$ limit from the collinear configuration trispectrum that considers the full mode-coupling integral over all angular terms is similar to the limit above from case I: only $s$-independent angular terms for any equal-sided configuration.

\section{Limits from Scalar Anisotropic Stress}

The trispectrum from magnetic scalar anisotropic stress Eq. (48) was found to be $10^{10}$ times larger than the trispectrum from magnetic energy density. Comparing it with the trispectrum from inflationary perturbations (Eq 67) gives a much stronger magnetic field constraint of

$$
B_{0} \lesssim 0.9 \mathrm{nG}
$$

using the positive-sided limit $\tau_{N L}<2,800$ from the Planck 2013 data release [61]. 
This $B_{0} \lesssim 0.9 \mathrm{nG}$ limit is over two and a half times as strong as the $B_{0}$ limit $(2.4 \mathrm{nG})$ obtained from the $\Pi_{B}$ bispectrum [59]. In addition, for those theories of inflation, which lead to $\tau_{N L}=\left(6 / 5 f_{N L}\right)^{2}$ we could perhaps use the relatively tighter limits on $f_{N L}$. The two-sigma limits on $f_{N L}^{l o c}$ are $-8.9<f_{N L}^{l o c}<14.3$, obtained from searching for the CMB primordial bispectrum signal in Planck 2013 data [61]. This gives a primordial magnetic field limit of

$$
B_{0} \lesssim 0.7 \mathrm{nG}
$$

for both the negative and positive $f_{N L}^{l o c}$ limits separately. We employ the local configuration $f_{N L}$ limits as the uncertainties $\sigma_{f_{N L}}$ in the other orthogonal and equilateral configurations are about an order of magnitude larger.

\section{Limits from Scalar Anisotropic Stress - Flat-Sky}

We can also compare the flat-sky calculation of the scalar anisotropic stress trispectrum to the trispectrum from inflationary perturbations (Eq. 67) and obtain magnetic field limits using the negative-sided limit of $\left|\tau_{N L}\right|<2,800$ to get

$$
B_{0} \lesssim 0.6-0.8 \mathrm{nG}
$$

The range of magnetic field upper limits reflects the range of $\sigma$ values (-84.6 to -14.2) in Table (III) for different configurations of the flat-sky trispectrum. As before, we may again consider those theories of inflation which lead to $\tau_{N L}=$ $\left(6 / 5 f_{N L}\right)^{2}$ and use the relatively tighter limits on $f_{N L}$, i.e $-8.9<f_{N L}^{l o c}<14.3$ [61] to place magnetic field upper limits of

$$
B_{0} \lesssim 0.4-0.6 \mathrm{nG}
$$

where we take the combined effect of the slightly different (positive and negative) limits for $f_{N L}$ as well as the range of values of $\sigma$ to arrive at the range of $B_{0}$ upper limits.

For magnetic scalar anisotropic stress, the flat-sky trispectra values give magnetic field upper limits that are slightly stronger but consistent with the sub-nanoGauss values derived from the full-sky trispectrum.

\section{CONCLUSIONS}

We have presented the full calculation for the CMB trispectrum sourced by primordial magnetic field scalar modes, first reported in our Letter [60]. In addition, we have calculated the scalar anisotropic stress trispectrum in the flat-sky limit. Together with recent improved observational constraints on primordial non-Gaussianity from the Planck mission 2013

\section{E. Limits from Inflationary Magnetic Curvature Mode}

Recently, Bonvin et al. [62, 89] have found a magnetic mode in the curvature perturbation that is present only when magnetic fields are generated at inflation. This magnetic mode is always scale-invariant and is absent when magnetogenesis occurs causally e.g. via a phase transition. This inflationary magnetic mode is seen to exist in addition to the compensated and passive modes and dominates over them in the CMB anisotropy. The ratio of the passive mode power spectrum to the new inflationary magnetic mode power spectrum is proportional to $\epsilon^{2}$ where $\epsilon \sim 10^{-2}$ is the inflationary slow-roll parameter. We calculate the passive to inflationary power spectrum ratio using the relation given between Equations (45) and (46) in Bonvin et al. [62, for $n \rightarrow-3$,

$$
\begin{aligned}
\frac{C_{l}^{\text {passive }}}{C_{l}^{\text {infl. mag. }}} \simeq & \epsilon^{2} \ln ^{2}\left(\frac{\eta_{*}}{\eta_{\nu}}\right)\left(\frac{\eta_{*}}{\eta_{0}}\right)^{2 n+6} \frac{\Gamma(-n-2)}{\Gamma\left(-n-\frac{3}{2}\right)} \\
& \times l^{2 n+6} \ln ^{2}\left(\frac{\eta_{*}}{\eta_{0}}\right),
\end{aligned}
$$

to find

$$
\frac{C_{l}^{\text {passive }}}{C_{l}^{\text {infl. mag. }}} \simeq 4.7 \times 10^{-5} .
$$

Now consider the magnetic CMB trispectrum sourced by this inflationary magnetic mode. We assume the trispectra ratio scales approximately as the power spectrum ratio squared and magnetic field constraint will come from one-eighth power of trispectra ratio. The magnetic field constraint is then found to be significantly stronger than from magnetic passive modes (i.e. scalar anisotropic stress $\left.\Pi_{B}\right)$ roughly by a factor $\approx(4.7 \times$ $\left.10^{-5}\right)^{-0.25} \approx 12$. The magnetic field upper limit from the inflationary magnetic mode CMB trispectrum is then

$$
B_{0} \lesssim 0.05 \mathrm{nG} \quad \text { i.e } \quad B_{0} \lesssim 50 \text { picoGauss. }
$$

For this inflationary magnetic mode, the trispectrum, as well as other CMB correlations, give magnetic field upper limits that are an order of magnitude stronger than those derived from the magnetic passive mode (scalar anisotropic stress) alone. Clearly, the new inflationary magnetic mode presented by Bonvin et al. [62] seems to place stronger constraints on primordial magnetic fields from its CMB correlations and we hope to return to this in greater detail in future work.

data, the magnetic scalar trispectrum enables us to place subnanoGauss upper limits on the strength primordial magnetic fields.

Magnetic energy density gives rise to a trispectrum of magnitude $\approx 10^{-29}$, for $s$-independent terms. Also, the collinear configuration trispectrum for energy density, including all angular terms, gives a result that is very similar to the case of $s$-independent terms for energy density. 
TABLE IV. Comparison of upper limits on primordial magnetic fields from magnetic mode contributions to the CMB power spectra, bispectra and trispectra (this work). We quote limits derived for close to scale-invariant magnetic fields and an early generation epoch (10 $\left.{ }^{14} \mathrm{GeV}\right)$ for magnetic passive modes.

\begin{tabular}{|c|c|c|c|}
\hline CMB Probe & Magnetic modes & Magnetic field upper limit $B_{0}(\mathrm{nG})$ & Reference \\
\hline Power Spectrum & scalar, vector \& tensor & 3.4 & [29] \\
\hline Bispectrum & energy density & $22^{\mathrm{a}}$ & [49] \\
\hline Bispectrum & scalar anisotropic stress & 2.4 & [59] \\
\hline Bispectrum & vector & 10 & {$[52]$} \\
\hline Bispectrum & tensor & 3.2 & {$[55]$} \\
\hline Trispectrum & energy density & 19 & this work \\
\hline Trispectrum & scalar anisotropic stress & 0.6 & this work \\
\hline Trispectrum & magnetic inflationary mode & 0.05 & this work; using [62] \\
\hline
\end{tabular}

a The magnetic field upper limit from [49] has been updated with the current values for $\mathcal{R}$ and current upper limit for $f_{N L}$

For magnetic scalar anisotropic stress, we find a trispectrum of magnitude $\approx 10^{-19}$, which is ten orders of magnitude larger than the magnetic energy density trispectrum. We also present an independent flat-sky limit calculation of this trispectrum with its angular structure that yields a slightly larger trispectrum of magnitude $\approx 10^{-18}$.

The magnetic energy density trispectrum allows us to place stronger upper limits on the primordial magnetic field compared to a similar calculation with the magnetic energy density bispectrum [49-51]. Further, the much larger trispectrum due to magnetic scalar anisotropic stress leads to the tightest constraint so far on large scale magnetic fields of $\sim 0.6 \mathrm{nG}$. This is approximately four times as strong as the corresponding upper limit from our previous bispectrum calculation $(\sim$ $2.4 \mathrm{nG}$ [ [59]. We note that the vector and tensor mode bispectra have been calculated numerically [52, 53, 55] and give magnetic field limits of $\sim 3-10 \mathrm{nG}$. Recently, polarization bispectra [56] constraints on magnetic fields have been forecast to be $\sim 2-3 \mathrm{nG}$ from expected Planck mission CMB polarization data. However, the scalar temperature trispectrum calculated in this work gives stronger magnetic fields constraints compared to the various kinds of bispectra that have been calculated (see Table( $(\mathrm{IV})$ ). The trispectrum's sensitivity can be illustrated by the magnetic to inflationary scalar trispectrum ratio, which is $\sim 10^{2}$ compared to $\sim 0.1$ for the ratio of magnetic to inflationary scalar bispectra (taking $f_{N L} \sim 10$ and $\left.B_{0} \sim 3 \mathrm{nG}\right)$.

We also note that the magnetic field upper limit at megaparsec scales derived from just the scalar mode magnetic CMB trispectrum is already several times better than the up- per limit from the magnetic CMB power spectrum combining scalar, vector and tensor modes: $3.4 \mathrm{nG}$ from Planck mission 2013 data [29] and ( 2-6 nG) from WMAP data [30-33]. Non-Gaussian correlations like the bispectrum and especially the trispectrum are better able to constrain primordial cosmological magnetic fields than the $\mathrm{CMB}$ power spectrum.

Finally, we have utilized the recently uncovered magnetic inflationary mode [62] as a source for the CMB trispectrum. This new magnetic mode dominates over both energy density and scalar anisotropic stress and leads to an order of magnitude stronger constraint on the primordial magnetic field of $\sim$ $0.05 \mathrm{nG}$. Further detailed investigation of the role this magnetic mode can play in sourcing various CMB correlations will be important.

Table (IV] summarizes the current constraints on primordial magnetic fields derived from various probes using CMB anisotropies, Thus, the CMB trispectrum is a new and more powerful probe of large scale primordial magnetic fields in the Universe.

Future consideration of magnetic vector and tensor modes in the trispectrum is likely to give additional constraints on primordial magnetic fields. Further improvement in magnetic field constraints is also possible from better $\tau_{N L}$ constraints that may emerge from a detailed analysis of the full Planck mission data. 


\section{ACKNOWLEDGEMENTS}

PT and TRS would like to acknowledge the IUCAA Associateship Program as well as the facilities at the IUCAA Resource Center, University of Delhi. PT would like to acknowledge support from Sri Venkateswara College, University of Delhi, in pursuing this work. TRS acknowledges support from CSIR India via grant-in-aid no. 03(1187)/11/EMR-II.

\section{Appendix: A}

In this Appendix we present the complete expressions for all angular terms generated by the sixteen operators present in the four-point correlation of magnetic anisotropic stress $\left\langle\Pi_{B}\left(\boldsymbol{k}_{1}\right) \Pi_{B}\left(\boldsymbol{k}_{2}\right) \Pi_{B}\left(\boldsymbol{k}_{3}\right) \Pi_{B}\left(\boldsymbol{k}_{4}\right)\right\rangle$ (Eq. 29. The extensive angular term expressions presented below have also been checked by taking an alternative order of contraction while calculating angular terms.

Each operator term $X$ from 1 to 16 generates its own separate angular term expression $\mathcal{F} \frac{\mathrm{x}}{(I)}$. When summed over all $X$ this yields the angular term expression $\mathcal{F}_{(I)}$, where $I$ takes values 1 to 6 in the six term mode-coupling integral $\left[\psi_{1234}\right]_{\Pi}$ below.

$$
\begin{aligned}
\psi_{1234}=\frac{8}{\left(8 \pi \rho_{0}\right)^{4}} \int d^{3} s M(s) M\left(\left|\boldsymbol{k}_{1}+\boldsymbol{s}\right|\right) & {\left[M\left(\left|\boldsymbol{k}_{1}+\boldsymbol{k}_{3}+\boldsymbol{s}\right|\right)\left(M\left(\left|\boldsymbol{k}_{2}-\boldsymbol{s}\right|\right) \mathcal{F}_{(1)}+M\left(\left|\boldsymbol{k}_{4}-\boldsymbol{s}\right|\right) \mathcal{F}_{(2)}\right)\right.} \\
& +M\left(\left|\boldsymbol{k}_{1}+\boldsymbol{k}_{2}+\boldsymbol{s}\right|\right)\left(M\left(\left|\boldsymbol{k}_{3}-\boldsymbol{s}\right|\right) \mathcal{F}_{(3)}+M\left(\left|\boldsymbol{k}_{4}-\boldsymbol{s}\right|\right) \mathcal{F}_{(4)}\right) \\
& \left.+M\left(\left|\boldsymbol{k}_{1}+\boldsymbol{k}_{4}+\boldsymbol{s}\right|\right)\left(M\left(\left|\boldsymbol{k}_{2}-\boldsymbol{s}\right|\right) \mathcal{F}_{(5)}+M\left(\left|\boldsymbol{k}_{3}-\boldsymbol{s}\right|\right) \mathcal{F}_{(6)}\right)\right]
\end{aligned}
$$

As seen in Eq. 29, the angular term expressions $\mathcal{F}$ generated by operators 2 to 5 will carry a prefactor of $(-3)$, angular term expressions generated by 6 to 11 will carry a prefactor of (9), angular term expressions generated by 12 to 15 will carry a prefactor of (-27) and the angular term expressions generated by 16 will have a prefactor of (81). For clarity, we suppress these prefactors while writing out the full angular term expressions below. The angles involved in these expressions have been defined earlier in Equations (11), (12) and in Table (I).

The angular terms for operator 1 are

$$
\begin{aligned}
& \mathcal{F} \frac{1}{(1)}=-1+\left(\alpha_{1}^{2}+\alpha_{2}^{2}+\alpha_{6}^{2}+\beta_{2}^{2}+\beta_{6}^{2}+\gamma_{6}^{2}\right)-\left(\alpha_{1} \alpha_{2} \beta_{2}+\alpha_{1} \alpha_{6} \beta_{6}+\alpha_{2} \alpha_{6} \gamma_{6}+\beta_{2} \beta_{6} \gamma_{6}\right)+\alpha_{1} \alpha_{2} \beta_{6} \gamma_{6} \\
& \mathcal{F} \frac{1}{(2)}=-1+\left(\alpha_{1}^{2}+\alpha_{4}^{2}+\alpha_{6}^{2}+\beta_{4}^{2}+\beta_{6}^{2}+\epsilon_{6}^{2}\right)-\left(\alpha_{1} \alpha_{4} \beta_{4}+\alpha_{1} \alpha_{6} \beta_{6}+\alpha_{4} \alpha_{6} \epsilon_{6}+\beta_{4} \beta_{6} \epsilon_{6}\right)+\alpha_{1} \alpha_{4} \beta_{6} \epsilon_{6} \\
& \mathcal{F} \frac{1}{(3)}=-1+\left(\alpha_{1}^{2}+\alpha_{3}^{2}+\alpha_{5}^{2}+\beta_{3}^{2}+\beta_{5}^{2}+\delta_{5}^{2}\right)-\left(\alpha_{1} \alpha_{3} \beta_{3}+\alpha_{1} \alpha_{5} \beta_{5}+\alpha_{3} \alpha_{5} \delta_{5}+\beta_{3} \beta_{5} \delta_{5}\right)+\alpha_{1} \alpha_{3} \beta_{5} \delta_{5} \\
& \mathcal{F} \frac{1}{(4)}=-1+\left(\alpha_{1}^{2}+\alpha_{4}^{2}+\alpha_{5}^{2}+\beta_{4}^{2}+\beta_{5}^{2}+\epsilon_{5}^{2}\right)-\left(\alpha_{1} \alpha_{4} \beta_{4}+\alpha_{1} \alpha_{5} \beta_{5}+\alpha_{4} \alpha_{5} \epsilon_{5}+\beta_{4} \beta_{5} \epsilon_{5}\right)+\alpha_{1} \alpha_{4} \beta_{5} \epsilon_{5} \\
& \mathcal{F} \frac{1}{(5)}=-1+\left(\alpha_{1}^{2}+\alpha_{2}^{2}+\alpha_{7}^{2}+\beta_{2}^{2}+\beta_{7}^{2}+\gamma_{7}^{2}\right)-\left(\alpha_{1} \alpha_{2} \beta_{2}+\alpha_{1} \alpha_{7} \beta_{7}+\alpha_{2} \alpha_{7} \gamma_{7}+\beta_{2} \beta_{7} \gamma_{7}\right)+\alpha_{1} \alpha_{2} \beta_{7} \gamma_{7} \\
& \mathcal{F} \frac{1}{(6)}=-1+\left(\alpha_{1}^{2}+\alpha_{3}^{2}+\alpha_{7}^{2}+\beta_{3}^{2}+\beta_{7}^{2}+\delta_{7}^{2}\right)-\left(\alpha_{1} \alpha_{3} \beta_{3}+\alpha_{1} \alpha_{7} \beta_{7}+\alpha_{3} \alpha_{7} \delta_{7}+\beta_{3} \beta_{7} \delta_{7}\right)+\alpha_{1} \alpha_{3} \beta_{7} \delta_{7} .
\end{aligned}
$$


The angular terms for operator 2 are

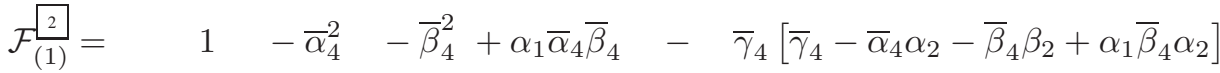

$$
\begin{aligned}
& -\bar{\lambda}_{4}\left[\bar{\lambda}_{4}-\bar{\alpha}_{4} \alpha_{6}-\bar{\beta}_{4} \beta_{6}+\alpha_{1} \bar{\alpha}_{4} \beta_{6}\right]+\bar{\gamma}_{4} \bar{\lambda}_{4}\left[\gamma_{6}-\alpha_{2} \alpha_{6}-\beta_{2} \beta_{6}+\alpha_{1} \alpha_{2} \beta_{6}\right]
\end{aligned}
$$

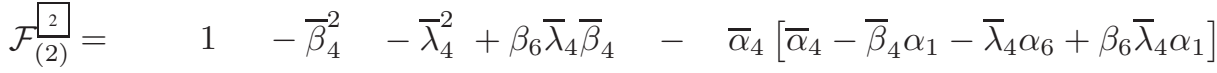

$$
\begin{aligned}
& -\bar{\epsilon}_{4}\left[\bar{\epsilon}_{4}-\bar{\beta}_{4} \beta_{4}-\bar{\lambda}_{4} \epsilon_{6}+\beta_{6} \bar{\beta}_{4} \epsilon_{6}\right]+\bar{\alpha}_{4} \bar{\epsilon}_{4}\left[\alpha_{4}-\alpha_{1} \beta_{4}-\alpha_{6} \epsilon_{6}+\beta_{6} \alpha_{1} \epsilon_{6}\right] \\
& \left.\mathcal{F} \frac{2}{(3)}=\quad \begin{array}{llll}
2 & -\bar{\alpha}_{4}^{2} & -\bar{\beta}_{4}^{2}+\alpha_{1} \bar{\alpha}_{4} \bar{\beta}_{4} & -\bar{\delta}_{4}
\end{array} \bar{\delta}_{4}-\bar{\alpha}_{4} \alpha_{3}-\bar{\beta}_{4} \beta_{3}+\alpha_{1} \bar{\beta}_{4} \alpha_{3}\right] \\
& -\bar{\kappa}_{4}\left[\bar{\kappa}_{4}-\bar{\alpha}_{4} \alpha_{5}-\bar{\beta}_{4} \beta_{5}+\alpha_{1} \bar{\alpha}_{4} \beta_{5}\right]+\bar{\delta}_{4} \bar{\kappa}_{4}\left[\delta_{5}-\alpha_{3} \alpha_{5}-\beta_{3} \beta_{5}+\alpha_{1} \alpha_{3} \beta_{5}\right]
\end{aligned}
$$

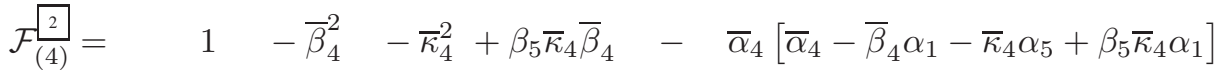

$$
\begin{aligned}
& -\bar{\epsilon}_{4}\left[\bar{\epsilon}_{4}-\bar{\beta}_{4} \beta_{4}-\bar{\kappa}_{4} \epsilon_{5}+\beta_{5} \bar{\beta}_{4} \epsilon_{5}\right]+\bar{\alpha}_{4} \bar{\epsilon}_{4}\left[\alpha_{4}-\alpha_{1} \beta_{4}-\alpha_{5} \epsilon_{5}+\beta_{5} \alpha_{1} \epsilon_{5}\right]
\end{aligned}
$$

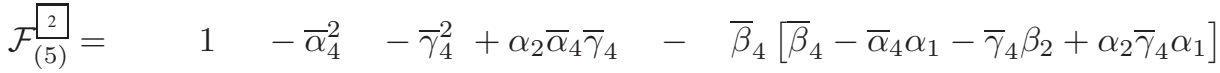

$$
\begin{aligned}
& -\bar{\chi}_{4}\left[\bar{\chi}_{4}-\bar{\alpha}_{4} \alpha_{7}-\bar{\gamma}_{4} \gamma_{7}+\alpha_{2} \bar{\alpha}_{4} \gamma_{7}\right]+\bar{\beta}_{4} \bar{\chi}_{4}\left[\beta_{7}-\alpha_{1} \alpha_{7}-\beta_{2} \gamma_{7}+\alpha_{2} \alpha_{1} \gamma_{7}\right] \\
& \mathcal{F} \frac{2}{(6)}=1-\bar{\alpha}_{4}^{2}-\bar{\delta}_{4}^{2}+\alpha_{3} \bar{\alpha}_{4} \bar{\delta}_{4}-\bar{\beta}_{4}\left[\bar{\beta}_{4}-\bar{\alpha}_{4} \alpha_{1}-\bar{\delta}_{4} \beta_{3}+\alpha_{3} \bar{\delta}_{4} \alpha_{1}\right] \\
& -\bar{\chi}_{4}\left[\bar{\chi}_{4}-\bar{\alpha}_{4} \alpha_{7}-\bar{\delta}_{4} \delta_{7}+\alpha_{3} \bar{\alpha}_{4} \delta_{7}\right]+\bar{\beta}_{4} \bar{\chi}_{4}\left[\beta_{7}-\alpha_{1} \alpha_{7}-\beta_{3} \delta_{7}+\alpha_{3} \alpha_{1} \delta_{7}\right] .
\end{aligned}
$$

The angular terms for operator 3 are

$$
\begin{aligned}
& \mathcal{F} \stackrel{3}{(1)}=\quad 1-\bar{\alpha}_{3}^{2}-\bar{\gamma}_{3}^{2}+\alpha_{2} \bar{\alpha}_{3} \bar{\gamma}_{3}-\bar{\beta}_{3}\left[\bar{\beta}_{3}-\bar{\alpha}_{3} \alpha_{1}-\bar{\gamma}_{3} \beta_{2}+\alpha_{2} \bar{\gamma}_{3} \alpha_{1}\right] \\
& -\bar{\lambda}_{3}\left[\bar{\lambda}_{3}-\bar{\alpha}_{3} \alpha_{6}-\bar{\gamma}_{3} \gamma_{6}+\alpha_{2} \bar{\alpha}_{3} \gamma_{6}\right]+\bar{\beta}_{3} \bar{\lambda}_{3}\left[\beta_{6}-\alpha_{1} \alpha_{6}-\beta_{2} \gamma_{6}+\alpha_{2} \alpha_{1} \gamma_{6}\right]
\end{aligned}
$$

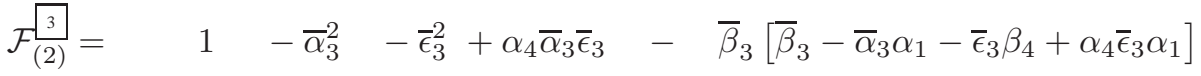

$$
\begin{aligned}
& -\bar{\lambda}_{3}\left[\bar{\lambda}_{3}-\bar{\alpha}_{3} \alpha_{6}-\bar{\epsilon}_{3} \epsilon_{6}+\alpha_{4} \bar{\alpha}_{3} \epsilon_{6}\right]+\bar{\beta}_{3} \bar{\lambda}_{3}\left[\beta_{6}-\alpha_{1} \alpha_{6}-\beta_{4} \epsilon_{6}+\alpha_{4} \alpha_{1} \epsilon_{6}\right] \\
& \mathcal{F} \frac{3}{(3)}=1-\bar{\beta}_{3}^{2}-\bar{\kappa}_{3}^{2}+\beta_{5} \bar{\beta}_{3} \bar{\kappa}_{3}-\bar{\alpha}_{3}\left[\bar{\alpha}_{3}-\bar{\beta}_{3} \alpha_{1}-\bar{\kappa}_{3} \alpha_{5}+\beta_{5} \bar{\kappa}_{3} \alpha_{1}\right] \\
& -\bar{\delta}_{3}\left[\bar{\delta}_{3}-\bar{\beta}_{3} \beta_{3}-\bar{\kappa}_{3} \delta_{5}+\beta_{5} \bar{\beta}_{3} \delta_{5}\right]+\bar{\alpha}_{3} \bar{\delta}_{3}\left[\alpha_{3}-\alpha_{1} \beta_{3}-\alpha_{5} \delta_{5}+\beta_{5} \alpha_{1} \delta_{5}\right]
\end{aligned}
$$

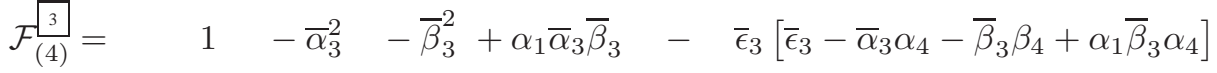

$$
\begin{aligned}
& -\bar{\kappa}_{3}\left[\bar{\kappa}_{3}-\bar{\alpha}_{3} \alpha_{5}-\bar{\beta}_{3} \beta_{5}+\alpha_{1} \bar{\alpha}_{3} \beta_{5}\right]+\bar{\epsilon}_{3} \bar{\kappa}_{3}\left[\epsilon_{5}-\alpha_{4} \alpha_{5}-\beta_{4} \beta_{5}+\alpha_{1} \alpha_{4} \beta_{5}\right] \\
& \mathcal{F} \frac{\sqrt[3]{(5)}}{=} \quad 1-\bar{\alpha}_{3}^{2}-\bar{\beta}_{3}^{2}+\alpha_{1} \bar{\alpha}_{3} \bar{\beta}_{3}-\bar{\gamma}_{3}\left[\bar{\gamma}_{3}-\bar{\alpha}_{3} \alpha_{2}-\bar{\beta}_{3} \beta_{2}+\alpha_{1} \bar{\beta}_{3} \alpha_{2}\right] \\
& -\bar{\chi}_{3}\left[\bar{\chi}_{3}-\bar{\alpha}_{3} \alpha_{7}-\bar{\beta}_{3} \beta_{7}+\alpha_{1} \bar{\alpha}_{3} \beta_{7}\right]+\bar{\gamma}_{3} \bar{\chi}_{3}\left[\gamma_{7}-\alpha_{2} \alpha_{7}-\beta_{2} \beta_{7}+\alpha_{1} \alpha_{2} \beta_{7}\right] \\
& \mathcal{F}_{(6)}^{-3}=1-\bar{\beta}_{3}^{2}-\bar{\chi}_{3}^{2}+\beta_{7} \bar{\beta}_{3} \bar{\chi}_{3}-\bar{\alpha}_{3}\left[\bar{\alpha}_{3}-\bar{\beta}_{3} \alpha_{1}-\bar{\chi}_{3} \alpha_{7}+\beta_{7} \bar{\chi}_{3} \alpha_{1}\right] \\
& -\bar{\delta}_{3}\left[\bar{\delta}_{3}-\bar{\beta}_{3} \beta_{3}-\bar{\chi}_{3} \delta_{7}+\beta_{7} \bar{\beta}_{3} \delta_{7}\right]+\bar{\alpha}_{3} \bar{\delta}_{3}\left[\alpha_{3}-\alpha_{1} \beta_{3}-\alpha_{7} \delta_{7}+\beta_{7} \alpha_{1} \delta_{7}\right] \text {. }
\end{aligned}
$$


The angular terms for operator 4 are

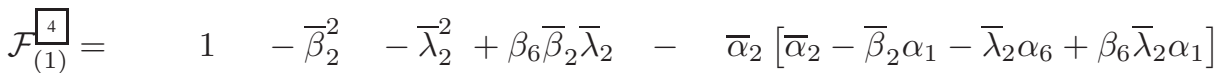

$$
\begin{aligned}
& -\bar{\gamma}_{2}\left[\bar{\gamma}_{2}-\bar{\beta}_{2} \beta_{2}-\bar{\lambda}_{2} \gamma_{6}+\beta_{6} \bar{\beta}_{2} \gamma_{6}\right]+\bar{\alpha}_{2} \bar{\gamma}_{2}\left[\alpha_{2}-\alpha_{1} \beta_{2}-\alpha_{6} \gamma_{6}+\beta_{6} \alpha_{1} \gamma_{6}\right]
\end{aligned}
$$

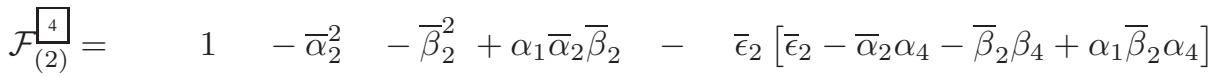

$$
\begin{aligned}
& -\bar{\lambda}_{2}\left[\bar{\lambda}_{2}-\bar{\alpha}_{2} \alpha_{6}-\bar{\beta}_{2} \beta_{6}+\alpha_{1} \bar{\alpha}_{2} \beta_{6}\right]+\bar{\epsilon}_{2} \bar{\lambda}_{2}\left[\epsilon_{6}-\alpha_{4} \alpha_{6}-\beta_{4} \beta_{6}+\alpha_{1} \alpha_{4} \beta_{6}\right]
\end{aligned}
$$



$$
\begin{aligned}
& -\bar{\kappa}_{2}\left[\bar{\kappa}_{2}-\bar{\alpha}_{2} \alpha_{5}-\bar{\delta}_{2} \delta_{5}+\alpha_{3} \bar{\alpha}_{2} \delta_{5}\right]+\bar{\beta}_{2} \bar{\kappa}_{2}\left[\beta_{5}-\alpha_{1} \alpha_{5}-\beta_{3} \delta_{5}+\alpha_{3} \alpha_{1} \delta_{5}\right] \\
& \mathcal{F} \underset{(4)}{-4}=1-\bar{\alpha}_{2}^{2}-\bar{\epsilon}_{2}^{2}+\alpha_{4} \bar{\alpha}_{2} \bar{\epsilon}_{2}-\bar{\beta}_{2}\left[\bar{\beta}_{2}-\bar{\alpha}_{2} \alpha_{1}-\bar{\epsilon}_{2} \beta_{4}+\alpha_{4} \bar{\epsilon}_{2} \alpha_{1}\right] \\
& -\bar{\kappa}_{2}\left[\bar{\kappa}_{2}-\bar{\alpha}_{2} \alpha_{5}-\bar{\epsilon}_{2} \epsilon_{5}+\alpha_{4} \bar{\alpha}_{2} \epsilon_{5}\right]+\bar{\beta}_{2} \bar{\kappa}_{2}\left[\beta_{5}-\alpha_{1} \alpha_{5}-\beta_{4} \epsilon_{5}+\alpha_{4} \alpha_{1} \epsilon_{5}\right] \\
& \mathcal{F}_{(5)}^{-4}=1-\bar{\beta}_{2}^{2}-\bar{\chi}_{2}^{2}+\beta_{7} \bar{\beta}_{2} \bar{\chi}_{2}-\bar{\alpha}_{2}\left[\bar{\alpha}_{2}-\bar{\beta}_{2} \alpha_{1}-\bar{\chi}_{2} \alpha_{7}+\beta_{7} \bar{\chi}_{2} \alpha_{1}\right] \\
& -\bar{\gamma}_{2}\left[\bar{\gamma}_{2}-\bar{\beta}_{2} \beta_{2}-\bar{\chi}_{2} \gamma_{7}+\beta_{7} \bar{\beta}_{2} \gamma_{7}\right]+\bar{\alpha}_{2} \bar{\gamma}_{2}\left[\alpha_{2}-\alpha_{1} \beta_{2}-\alpha_{7} \gamma_{7}+\beta_{7} \alpha_{1} \gamma_{7}\right] \\
& \mathcal{F}-\frac{4}{(6)}=1-\bar{\alpha}_{2}^{2}-\bar{\beta}_{2}^{2}+\alpha_{1} \bar{\alpha}_{2} \bar{\beta}_{2}-\bar{\delta}_{2}\left[\bar{\delta}_{2}-\bar{\alpha}_{2} \alpha_{3}-\bar{\beta}_{2} \beta_{3}+\alpha_{1} \bar{\beta}_{2} \alpha_{3}\right] \\
& -\bar{\chi}_{2}\left[\bar{\chi}_{2}-\bar{\alpha}_{2} \alpha_{7}-\bar{\beta}_{2} \beta_{7}+\alpha_{1} \bar{\alpha}_{2} \beta_{7}\right]+\bar{\delta}_{2} \bar{\chi}_{2}\left[\delta_{7}-\alpha_{3} \alpha_{7}-\beta_{3} \beta_{7}+\alpha_{1} \alpha_{3} \beta_{7}\right] .
\end{aligned}
$$

The angular terms for operator 5 are

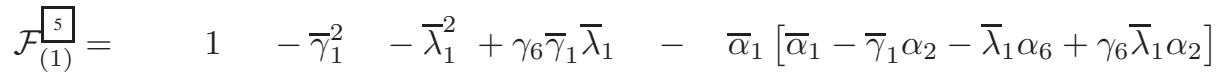

$$
\begin{aligned}
& -\bar{\beta}_{1}\left[\bar{\beta}_{1}-\bar{\gamma}_{1} \beta_{2}-\bar{\lambda}_{1} \beta_{6}+\gamma_{6} \bar{\gamma}_{1} \beta_{6}\right]+\bar{\alpha}_{1} \bar{\beta}_{1}\left[\alpha_{1}-\alpha_{2} \beta_{2}-\alpha_{6} \beta_{6}+\gamma_{6} \alpha_{2} \beta_{6}\right]
\end{aligned}
$$



$$
\begin{aligned}
& -\bar{\beta}_{1}\left[\bar{\beta}_{1}-\bar{\epsilon}_{1} \beta_{4}-\bar{\lambda}_{1} \beta_{6}+\epsilon_{6} \bar{\epsilon}_{1} \beta_{6}\right]+\bar{\alpha}_{1} \bar{\beta}_{1}\left[\alpha_{1}-\alpha_{4} \beta_{4}-\alpha_{6} \beta_{6}+\epsilon_{6} \alpha_{4} \beta_{6}\right] \\
& \left.\mathcal{F} \frac{5}{(3)}=\quad \begin{array}{lllll}
5 & -\bar{\delta}_{1}^{2} & -\bar{\kappa}_{1}^{2}+\delta_{5} \bar{\delta}_{1} \bar{\kappa}_{1} & - & \bar{\alpha}_{1}
\end{array} \bar{\alpha}_{1}-\bar{\delta}_{1} \alpha_{3}-\bar{\kappa}_{1} \alpha_{5}+\delta_{5} \bar{\kappa}_{1} \alpha_{3}\right] \\
& -\bar{\beta}_{1}\left[\bar{\beta}_{1}-\bar{\delta}_{1} \beta_{3}-\bar{\kappa}_{1} \beta_{5}+\delta_{5} \bar{\delta}_{1} \beta_{5}\right]+\bar{\alpha}_{1} \bar{\beta}_{1}\left[\alpha_{1}-\alpha_{3} \beta_{3}-\alpha_{5} \beta_{5}+\delta_{5} \alpha_{3} \beta_{5}\right] \\
& \mathcal{F} \frac{5}{(4)}=\quad \begin{array}{lllll}
5 & -\bar{\epsilon}_{1}^{2} & -\bar{\kappa}_{1}^{2}+\epsilon_{5} \bar{\epsilon}_{1} \bar{\kappa}_{1} & - & \bar{\alpha}_{1}\left[\bar{\alpha}_{1}-\bar{\epsilon}_{1} \alpha_{4}-\bar{\kappa}_{1} \alpha_{5}+\epsilon_{5} \bar{\kappa}_{1} \alpha_{4}\right]
\end{array} \\
& -\bar{\beta}_{1}\left[\bar{\beta}_{1}-\bar{\epsilon}_{1} \beta_{4}-\bar{\kappa}_{1} \beta_{5}+\epsilon_{5} \bar{\epsilon}_{1} \beta_{5}\right]+\bar{\alpha}_{1} \bar{\beta}_{1}\left[\alpha_{1}-\alpha_{4} \beta_{4}-\alpha_{5} \beta_{5}+\epsilon_{5} \alpha_{4} \beta_{5}\right]
\end{aligned}
$$

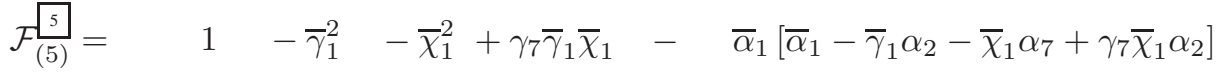

$$
\begin{aligned}
& -\bar{\beta}_{1}\left[\bar{\beta}_{1}-\bar{\gamma}_{1} \beta_{2}-\bar{\chi}_{1} \beta_{7}+\gamma_{7} \bar{\gamma}_{1} \beta_{7}\right]+\bar{\alpha}_{1} \bar{\beta}_{1}\left[\alpha_{1}-\alpha_{2} \beta_{2}-\alpha_{7} \beta_{7}+\gamma_{7} \alpha_{2} \beta_{7}\right]
\end{aligned}
$$

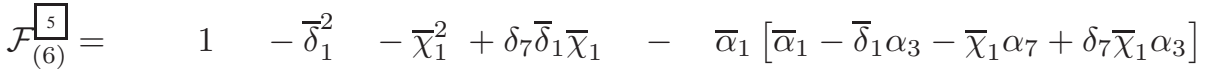

$$
\begin{aligned}
& -\bar{\beta}_{1}\left[\bar{\beta}_{1}-\bar{\delta}_{1} \beta_{3}-\bar{\chi}_{1} \beta_{7}+\delta_{7} \bar{\delta}_{1} \beta_{7}\right]+\bar{\alpha}_{1} \bar{\beta}_{1}\left[\alpha_{1}-\alpha_{3} \beta_{3}-\alpha_{7} \beta_{7}+\delta_{7} \alpha_{3} \beta_{7}\right] .
\end{aligned}
$$

The angular terms for operator 6 are

$$
\begin{aligned}
& \mathcal{F} \frac{\sqrt[6]{(1)}}{=}=\left[\theta_{34}-\bar{\beta}_{3} \bar{\beta}_{4}-\bar{\gamma}_{4}\left(\bar{\gamma}_{3}-\beta_{2} \bar{\beta}_{3}\right)-\bar{\alpha}_{4}\left(\bar{\alpha}_{3}-\alpha_{1} \bar{\beta}_{3}\right)+\bar{\gamma}_{4} \alpha_{2}\left(\bar{\alpha}_{3}-\alpha_{1} \bar{\beta}_{3}\right)\right]\left(\theta_{34}-\bar{\lambda}_{3} \bar{\lambda}_{4}\right) \\
& \mathcal{F} \frac{6}{(2)}=\left(\theta_{34}-\bar{\alpha}_{3} \bar{\alpha}_{4}-\bar{\beta}_{3} \bar{\beta}_{4}+\alpha_{1} \bar{\alpha}_{4} \bar{\beta}_{3}\right)\left(\theta_{34}-\bar{\epsilon}_{3} \bar{\epsilon}_{4}-\bar{\lambda}_{3} \bar{\lambda}_{4}+\epsilon_{6} \bar{\epsilon}_{4} \bar{\lambda}_{3}\right) \\
& \mathcal{F} \frac{\sqrt[6]{(3)}}{6}=\left[\theta_{34}-\bar{\alpha}_{3} \bar{\alpha}_{4}-\bar{\kappa}_{4}\left(\bar{\kappa}_{3}-\alpha_{5} \bar{\alpha}_{3}\right)-\bar{\beta}_{4}\left(\bar{\beta}_{3}-\alpha_{1} \bar{\alpha}_{3}\right)+\bar{\kappa}_{4} \beta_{5}\left(\bar{\beta}_{3}-\alpha_{1} \bar{\alpha}_{3}\right)\right]\left(\theta_{34}-\bar{\delta}_{3} \bar{\delta}_{4}\right) \\
& \mathcal{F} \frac{6}{(4)}=\left[\theta_{34}-\bar{\alpha}_{3} \bar{\alpha}_{4}-\bar{\kappa}_{3}\left(\bar{\kappa}_{4}-\alpha_{5} \bar{\alpha}_{4}\right)-\bar{\beta}_{3}\left(\bar{\beta}_{4}-\alpha_{1} \bar{\alpha}_{4}\right)+\bar{\kappa}_{3} \beta_{5}\left(\bar{\beta}_{4}-\alpha_{1} \bar{\alpha}_{4}\right)\right]\left(\theta_{34}-\bar{\epsilon}_{3} \bar{\epsilon}_{4}\right) \\
& \mathcal{F} \frac{6}{(5)}=\left[\theta_{34}-\bar{\beta}_{3} \bar{\beta}_{4}-\bar{\gamma}_{3}\left(\bar{\gamma}_{4}-\beta_{2} \bar{\beta}_{4}\right)-\bar{\alpha}_{3}\left(\bar{\alpha}_{4}-\alpha_{1} \bar{\beta}_{4}\right)+\bar{\gamma}_{3} \alpha_{2}\left(\bar{\alpha}_{4}-\alpha_{1} \bar{\beta}_{4}\right)\right]\left(\theta_{34}-\bar{\chi}_{3} \bar{\chi}_{4}\right) \\
& \mathcal{F} \frac{6}{(6)}=\left(\theta_{34}-\bar{\alpha}_{3} \bar{\alpha}_{4}-\bar{\beta}_{3} \bar{\beta}_{4}+\alpha_{1} \bar{\alpha}_{3} \bar{\beta}_{4}\right)\left(\theta_{34}-\bar{\delta}_{3} \bar{\delta}_{4}-\bar{\chi}_{3} \bar{\chi}_{4}+\delta_{7} \bar{\delta}_{3} \bar{\chi}_{4}\right) .
\end{aligned}
$$


The angular terms for operator 7 are

$$
\begin{aligned}
& \mathcal{F} \underset{(1)}{?}=\left[\theta_{24}-\bar{\alpha}_{2} \bar{\alpha}_{4}-\bar{\lambda}_{4}\left(\bar{\lambda}_{2}-\alpha_{6} \bar{\alpha}_{2}\right)-\bar{\beta}_{4}\left(\bar{\beta}_{2}-\alpha_{1} \bar{\alpha}_{2}\right)+\bar{\lambda}_{4} \beta_{6}\left(\bar{\beta}_{2}-\alpha_{1} \bar{\alpha}_{2}\right)\right]\left(\theta_{24}-\bar{\gamma}_{2} \bar{\gamma}_{4}\right) \\
& \mathcal{F} \frac{7}{(2)}=\left[\theta_{24}-\bar{\alpha}_{2} \bar{\alpha}_{4}-\bar{\lambda}_{2}\left(\bar{\lambda}_{4}-\alpha_{6} \bar{\alpha}_{4}\right)-\bar{\beta}_{2}\left(\bar{\beta}_{4}-\alpha_{1} \bar{\alpha}_{4}\right)+\bar{\lambda}_{2} \beta_{6}\left(\bar{\beta}_{4}-\alpha_{1} \bar{\alpha}_{4}\right)\right]\left(\theta_{24}-\bar{\epsilon}_{2} \bar{\epsilon}_{4}\right) \\
& \mathcal{F} \frac{7}{(3)}=\left[\theta_{24}-\bar{\beta}_{2} \bar{\beta}_{4}-\bar{\delta}_{4}\left(\bar{\delta}_{2}-\beta_{3} \bar{\beta}_{2}\right)-\bar{\alpha}_{4}\left(\bar{\alpha}_{2}-\alpha_{1} \bar{\beta}_{2}\right)+\bar{\delta}_{4} \alpha_{3}\left(\bar{\alpha}_{2}-\alpha_{1} \bar{\beta}_{2}\right)\right]\left(\theta_{24}-\bar{\kappa}_{2} \bar{\kappa}_{4}\right) \\
& \mathcal{F} \underset{(4)}{7}=\left(\theta_{24}-\bar{\alpha}_{2} \bar{\alpha}_{4}-\bar{\beta}_{2} \bar{\beta}_{4}+\alpha_{1} \bar{\alpha}_{4} \bar{\beta}_{2}\right)\left(\theta_{24}-\bar{\epsilon}_{2} \bar{\epsilon}_{4}-\bar{\kappa}_{2} \bar{\kappa}_{4}+\epsilon_{5} \bar{\epsilon}_{4} \bar{\kappa}_{2}\right) \\
& \mathcal{F} \frac{7}{(5)}=\left(\theta_{24}-\bar{\alpha}_{2} \bar{\alpha}_{4}-\bar{\beta}_{2} \bar{\beta}_{4}+\alpha_{1} \bar{\alpha}_{2} \bar{\beta}_{4}\right)\left(\theta_{24}-\bar{\gamma}_{2} \bar{\gamma}_{4}-\bar{\chi}_{2} \bar{\chi}_{4}+\gamma_{7} \bar{\gamma}_{2} \bar{\chi}_{4}\right) \\
& \mathcal{F} \frac{\square}{(6)}=\left[\theta_{24}-\bar{\beta}_{2} \bar{\beta}_{4}-\bar{\delta}_{2}\left(\bar{\delta}_{4}-\beta_{3} \bar{\beta}_{4}\right)-\bar{\alpha}_{2}\left(\bar{\alpha}_{4}-\alpha_{1} \bar{\beta}_{4}\right)+\bar{\delta}_{2} \alpha_{3}\left(\bar{\alpha}_{4}-\alpha_{1} \bar{\beta}_{4}\right)\right]\left(\theta_{24}-\bar{\chi}_{2} \bar{\chi}_{4}\right) \text {. }
\end{aligned}
$$

The angular terms for operator 8 are

$$
\begin{aligned}
& \mathcal{F} \frac{\sqrt[8]{(1)}}{=}=\left(\theta_{23}-\bar{\alpha}_{2} \bar{\alpha}_{3}-\bar{\beta}_{2} \bar{\beta}_{3}+\alpha_{1} \bar{\alpha}_{2} \bar{\beta}_{3}\right)\left(\theta_{23}-\bar{\gamma}_{2} \bar{\gamma}_{3}-\bar{\lambda}_{2} \bar{\lambda}_{3}+\gamma_{6} \bar{\gamma}_{2} \bar{\lambda}_{3}\right) \\
& \mathcal{F} \underset{(2)}{-\frac{8}{(2)}}=\left[\theta_{23}-\bar{\beta}_{2} \bar{\beta}_{3}-\bar{\epsilon}_{2}\left(\bar{\epsilon}_{3}-\beta_{4} \bar{\beta}_{3}\right)-\bar{\alpha}_{2}\left(\bar{\alpha}_{3}-\alpha_{1} \bar{\beta}_{3}\right)+\bar{\epsilon}_{2} \alpha_{4}\left(\bar{\alpha}_{3}-\alpha_{1} \bar{\beta}_{3}\right)\right]\left(\theta_{23}-\bar{\lambda}_{2} \bar{\lambda}_{3}\right) \\
& \mathcal{F} \frac{8}{(3)}=\left(\theta_{23}-\bar{\alpha}_{2} \bar{\alpha}_{3}-\bar{\beta}_{2} \bar{\beta}_{3}+\alpha_{1} \bar{\alpha}_{3} \bar{\beta}_{2}\right)\left(\theta_{23}-\bar{\delta}_{2} \bar{\delta}_{3}-\bar{\kappa}_{2} \bar{\kappa}_{3}+\delta_{5} \bar{\delta}_{3} \bar{\kappa}_{2}\right) \\
& \mathcal{F} \mathcal{F}_{(4)}^{8}=\left[\theta_{23}-\bar{\beta}_{2} \bar{\beta}_{3}-\bar{\epsilon}_{3}\left(\bar{\epsilon}_{2}-\beta_{4} \bar{\beta}_{2}\right)-\bar{\alpha}_{3}\left(\bar{\alpha}_{2}-\alpha_{1} \bar{\beta}_{2}\right)+\bar{\epsilon}_{3} \alpha_{4}\left(\bar{\alpha}_{2}-\alpha_{1} \bar{\beta}_{2}\right)\right]\left(\theta_{23}-\bar{\kappa}_{2} \bar{\kappa}_{3}\right) \\
& \mathcal{F} \frac{8}{(5)}=\left[\theta_{23}-\bar{\alpha}_{2} \bar{\alpha}_{3}-\bar{\chi}_{3}\left(\bar{\chi}_{2}-\alpha_{7} \bar{\alpha}_{2}\right)-\bar{\beta}_{3}\left(\bar{\beta}_{2}-\alpha_{1} \bar{\alpha}_{2}\right)+\bar{\chi}_{3} \beta_{7}\left(\bar{\beta}_{2}-\alpha_{1} \bar{\alpha}_{2}\right)\right]\left(\theta_{23}-\bar{\gamma}_{2} \bar{\gamma}_{3}\right) \\
& \mathcal{F} \frac{8}{(6)}=\left[\theta_{23}-\bar{\alpha}_{2} \bar{\alpha}_{3}-\bar{\chi}_{2}\left(\bar{\chi}_{3}-\alpha_{7} \bar{\alpha}_{3}\right)-\bar{\beta}_{2}\left(\bar{\beta}_{3}-\alpha_{1} \bar{\alpha}_{3}\right)+\bar{\chi}_{2} \beta_{7}\left(\bar{\beta}_{3}-\alpha_{1} \bar{\alpha}_{3}\right)\right]\left(\theta_{23}-\bar{\delta}_{2} \bar{\delta}_{3}\right) .
\end{aligned}
$$

The angular terms for operator 9 are

$$
\begin{aligned}
& \mathcal{F} \frac{9}{(1)}=\left(\theta_{14}-\bar{\alpha}_{1} \bar{\alpha}_{4}-\bar{\gamma}_{1} \bar{\gamma}_{4}+\alpha_{2} \bar{\alpha}_{1} \bar{\gamma}_{4}\right)\left(\theta_{14}-\bar{\beta}_{1} \bar{\beta}_{4}-\bar{\lambda}_{1} \bar{\lambda}_{4}+\alpha_{6} \bar{\beta}_{1} \bar{\lambda}_{4}\right) \\
& \mathcal{F} \frac{9}{(2)}=\left[\theta_{14}-\bar{\beta}_{1} \bar{\beta}_{4}-\bar{\epsilon}_{4}\left(\bar{\epsilon}_{1}-\beta_{4} \bar{\beta}_{1}\right)-\bar{\lambda}_{4}\left(\bar{\lambda}_{1}-\beta_{6} \bar{\beta}_{1}\right)+\bar{\epsilon}_{4} \epsilon_{6}\left(\bar{\lambda}_{1}-\beta_{6} \bar{\beta}_{1}\right)\right]\left(\theta_{14}-\bar{\alpha}_{1} \bar{\alpha}_{4}\right) \\
& \mathcal{F} \frac{9}{(3)}=\left(\theta_{14}-\bar{\alpha}_{1} \bar{\alpha}_{4}-\bar{\delta}_{1} \bar{\delta}_{4}+\alpha_{2} \bar{\alpha}_{1} \bar{\delta}_{4}\right)\left(\theta_{14}-\bar{\beta}_{1} \bar{\beta}_{4}-\bar{\kappa}_{1} \bar{\kappa}_{4}+\beta_{5} \bar{\beta}_{1} \bar{\kappa}_{4}\right) \\
& \mathcal{F} \mathcal{P} \frac{9}{(4)}=\left[\theta_{14}-\bar{\beta}_{1} \bar{\beta}_{4}-\bar{\epsilon}_{4}\left(\bar{\epsilon}_{1}-\beta_{4} \bar{\beta}_{1}\right)-\bar{\kappa}_{4}\left(\bar{\kappa}_{1}-\beta_{5} \bar{\beta}_{1}\right)+\bar{\epsilon}_{4} \epsilon_{5}\left(\bar{\kappa}_{1}-\beta_{5} \bar{\beta}_{1}\right)\right]\left(\theta_{14}-\bar{\alpha}_{1} \bar{\alpha}_{4}\right) \\
& \mathcal{F} \frac{9}{(5)}=\left[\theta_{14}-\bar{\alpha}_{1} \bar{\alpha}_{4}-\bar{\chi}_{4}\left(\bar{\chi}_{1}-\alpha_{7} \bar{\alpha}_{1}\right)-\bar{\gamma}_{4}\left(\bar{\gamma}_{1}-\alpha_{2} \bar{\alpha}_{1}\right)+\bar{\chi}_{4} \gamma_{7}\left(\bar{\gamma}_{1}-\alpha_{2} \bar{\alpha}_{1}\right)\right]\left(\theta_{14}-\bar{\beta}_{1} \bar{\beta}_{4}\right) \\
& \mathcal{F} \frac{9}{(6)}=\left[\theta_{14}-\bar{\alpha}_{1} \bar{\alpha}_{4}-\bar{\chi}_{4}\left(\bar{\chi}_{1}-\alpha_{7} \bar{\alpha}_{1}\right)-\bar{\delta}_{4}\left(\bar{\delta}_{1}-\alpha_{3} \bar{\alpha}_{1}\right)+\bar{\chi}_{4} \delta_{7}\left(\bar{\delta}_{1}-\alpha_{3} \bar{\alpha}_{1}\right)\right]\left(\theta_{14}-\bar{\beta}_{1} \bar{\beta}_{4}\right) .
\end{aligned}
$$

The angular terms for operator 10 are

$$
\begin{aligned}
& \mathcal{F}_{(1)}^{\stackrel{10}{(1)}}=\left[\theta_{13}-\bar{\alpha}_{1} \bar{\alpha}_{3}-\bar{\lambda}_{3}\left(\bar{\lambda}_{1}-\alpha_{6} \bar{\alpha}_{1}\right)-\bar{\gamma}_{3}\left(\bar{\gamma}_{1}-\alpha_{2} \bar{\alpha}_{1}\right)+\bar{\lambda}_{3} \gamma_{6}\left(\bar{\gamma}_{1}-\alpha_{2} \bar{\alpha}_{1}\right)\right]\left(\theta_{13}-\bar{\beta}_{1} \bar{\beta}_{3}\right) \\
& \mathcal{F}_{(2)}^{10}=\left[\theta_{13}-\bar{\alpha}_{1} \bar{\alpha}_{3}-\bar{\lambda}_{3}\left(\bar{\lambda}_{1}-\alpha_{6} \bar{\alpha}_{1}\right)-\bar{\epsilon}_{3}\left(\bar{\epsilon}_{1}-\alpha_{4} \bar{\alpha}_{1}\right)+\bar{\lambda}_{3} \epsilon_{6}\left(\bar{\epsilon}_{1}-\alpha_{4} \bar{\alpha}_{1}\right)\right]\left(\theta_{13}-\bar{\beta}_{1} \bar{\beta}_{3}\right) \\
& \mathcal{F} \frac{10}{(3)}=\left[\theta_{13}-\bar{\beta}_{1} \bar{\beta}_{3}-\bar{\delta}_{3}\left(\bar{\delta}_{1}-\beta_{3} \bar{\beta}_{1}\right)-\bar{\kappa}_{3}\left(\bar{\kappa}_{1}-\beta_{5} \bar{\beta}_{1}\right)+\bar{\delta}_{3} \delta_{5}\left(\bar{\kappa}_{1}-\beta_{5} \bar{\beta}_{1}\right)\right]\left(\theta_{13}-\bar{\alpha}_{1} \bar{\alpha}_{3}\right) \\
& \mathcal{F} \frac{100}{(4)}=\left(\theta_{13}-\bar{\alpha}_{1} \bar{\alpha}_{3}-\bar{\epsilon}_{1} \bar{\epsilon}_{3}+\alpha_{4} \bar{\alpha}_{1} \bar{\epsilon}_{3}\right)\left(\theta_{13}-\bar{\beta}_{1} \bar{\beta}_{3}-\bar{\kappa}_{1} \bar{\kappa}_{3}+\beta_{5} \bar{\beta}_{1} \bar{\kappa}_{3}\right) \\
& \mathcal{F} \frac{10}{(5)}=\left(\theta_{13}-\bar{\alpha}_{1} \bar{\alpha}_{3}-\bar{\gamma}_{1} \bar{\gamma}_{3}+\alpha_{2} \bar{\alpha}_{1} \bar{\gamma}_{3}\right)\left(\theta_{13}-\bar{\beta}_{1} \bar{\beta}_{3}-\bar{\chi}_{1} \bar{\chi}_{3}+\beta_{7} \bar{\beta}_{1} \bar{\chi}_{3}\right) \\
& \mathcal{F} \frac{10}{(6)}=\left[\theta_{13}-\bar{\beta}_{1} \bar{\beta}_{3}-\bar{\delta}_{3}\left(\bar{\delta}_{1}-\beta_{3} \bar{\beta}_{1}\right)-\bar{\chi}_{3}\left(\bar{\chi}_{1}-\beta_{7} \bar{\beta}_{1}\right)+\bar{\delta}_{3} \delta_{7}\left(\bar{\chi}_{1}-\beta_{7} \bar{\beta}_{1}\right)\right]\left(\theta_{13}-\bar{\alpha}_{1} \bar{\alpha}_{3}\right) .
\end{aligned}
$$


The angular terms for operator 11 are

$$
\begin{aligned}
& \mathcal{F} \frac{11}{(1)}=\left[\theta_{12}-\bar{\beta}_{1} \bar{\beta}_{2}-\bar{\gamma}_{2}\left(\bar{\gamma}_{1}-\beta_{2} \bar{\beta}_{1}\right)-\bar{\lambda}_{2}\left(\bar{\lambda}_{1}-\beta_{6} \bar{\beta}_{1}\right)+\bar{\gamma}_{2} \gamma_{6}\left(\bar{\lambda}_{1}-\beta_{6} \bar{\beta}_{1}\right)\right]\left(\theta_{12}-\bar{\alpha}_{1} \bar{\alpha}_{2}\right) \\
& \mathcal{F} \frac{11}{(2)}=\left(\theta_{12}-\bar{\alpha}_{1} \bar{\alpha}_{2}-\bar{\epsilon}_{1} \bar{\epsilon}_{2}+\alpha_{4} \bar{\alpha}_{1} \bar{\epsilon}_{2}\right)\left(\theta_{12}-\bar{\beta}_{1} \bar{\beta}_{2}-\bar{\lambda}_{1} \bar{\lambda}_{2}+\beta_{6} \bar{\beta}_{1} \bar{\lambda}_{2}\right) \\
& \mathcal{F} \frac{11}{(3)}=\left[\theta_{12}-\bar{\alpha}_{1} \bar{\alpha}_{2}-\bar{\kappa}_{2}\left(\bar{\kappa}_{1}-\alpha_{5} \bar{\alpha}_{1}\right)-\bar{\delta}_{2}\left(\bar{\delta}_{1}-\alpha_{3} \bar{\alpha}_{1}\right)+\bar{\kappa}_{2} \delta_{5}\left(\bar{\delta}_{1}-\alpha_{3} \bar{\alpha}_{1}\right)\right]\left(\theta_{12}-\bar{\beta}_{1} \bar{\beta}_{2}\right) \\
& \mathcal{F} \frac{\alpha_{(1)}}{(4)}=\left[\theta_{12}-\bar{\alpha}_{1} \bar{\alpha}_{2}-\bar{\kappa}_{2}\left(\bar{\kappa}_{1}-\alpha_{5} \bar{\alpha}_{1}\right)-\bar{\epsilon}_{2}\left(\bar{\epsilon}_{1}-\alpha_{4} \bar{\alpha}_{1}\right)+\bar{\kappa}_{2} \epsilon_{5}\left(\bar{\epsilon}_{1}-\alpha_{4} \bar{\alpha}_{1}\right)\right]\left(\theta_{12}-\bar{\beta}_{1} \bar{\beta}_{2}\right) \\
& \mathcal{F} \frac{11}{(5)}=\left[\theta_{12}-\bar{\beta}_{1} \bar{\beta}_{2}-\bar{\gamma}_{2}\left(\bar{\gamma}_{1}-\beta_{2} \bar{\beta}_{1}\right)-\bar{\chi}_{2}\left(\bar{\chi}_{1}-\beta_{7} \bar{\beta}_{1}\right)+\bar{\gamma}_{2} \gamma_{7}\left(\bar{\chi}_{1}-\beta_{7} \bar{\beta}_{1}\right)\right]\left(\theta_{12}-\bar{\alpha}_{1} \bar{\alpha}_{2}\right) \\
& \mathcal{F} \frac{11}{(6)}=\left(\theta_{12}-\bar{\alpha}_{1} \bar{\alpha}_{2}-\bar{\delta}_{1} \bar{\delta}_{2}+\alpha_{3} \bar{\alpha}_{1} \bar{\delta}_{2}\right)\left(\theta_{12}-\bar{\beta}_{1} \bar{\beta}_{2}-\bar{\chi}_{1} \bar{\chi}_{2}+\beta_{7} \bar{\beta}_{1} \bar{\chi}_{2}\right) .
\end{aligned}
$$

The angular terms for operator 12 are

$$
\begin{aligned}
& \mathcal{F} \frac{12}{(1)}=\left(\theta_{23}-\bar{\alpha}_{2} \bar{\alpha}_{3}-\bar{\beta}_{2} \bar{\beta}_{3}+\alpha_{1} \bar{\alpha}_{2} \bar{\beta}_{3}\right)\left(\theta_{24}-\bar{\gamma}_{2} \bar{\gamma}_{4}\right)\left(\theta_{34}-\bar{\lambda}_{3} \bar{\lambda}_{4}\right) \\
& \mathcal{F} \frac{12}{(2)}=\left(\theta_{34}-\bar{\alpha}_{3} \bar{\alpha}_{4}-\bar{\beta}_{3} \bar{\beta}_{4}+\alpha_{1} \bar{\alpha}_{4} \bar{\beta}_{3}\right)\left(\theta_{24}-\bar{\epsilon}_{2} \bar{\epsilon}_{4}\right)\left(\theta_{23}-\bar{\lambda}_{2} \bar{\lambda}_{3}\right) \\
& \mathcal{F} \frac{12}{(3)}=\left(\theta_{23}-\bar{\alpha}_{2} \bar{\alpha}_{3}-\bar{\beta}_{2} \bar{\beta}_{3}+\alpha_{1} \bar{\alpha}_{3} \bar{\beta}_{2}\right)\left(\theta_{34}-\bar{\delta}_{3} \bar{\delta}_{4}\right)\left(\theta_{24}-\bar{\kappa}_{2} \bar{\kappa}_{4}\right) \\
& \mathcal{F} \frac{12}{(4)}=\left(\theta_{24}-\bar{\alpha}_{2} \bar{\alpha}_{4}-\bar{\beta}_{2} \bar{\beta}_{4}+\alpha_{1} \bar{\alpha}_{4} \bar{\beta}_{2}\right)\left(\theta_{34}-\bar{\epsilon}_{3} \bar{\epsilon}_{4}\right)\left(\theta_{23}-\bar{\kappa}_{2} \bar{\kappa}_{3}\right) \\
& \mathcal{F} \frac{12}{(5)}=\left(\theta_{24}-\bar{\alpha}_{2} \bar{\alpha}_{4}-\bar{\beta}_{2} \bar{\beta}_{4}+\alpha_{1} \bar{\alpha}_{2} \bar{\beta}_{4}\right)\left(\theta_{23}-\bar{\gamma}_{2} \bar{\gamma}_{3}\right)\left(\theta_{34}-\bar{\chi}_{3} \bar{\chi}_{4}\right) \\
& \mathcal{F} \frac{12}{(6)}=\left(\theta_{34}-\bar{\alpha}_{3} \bar{\alpha}_{4}-\bar{\beta}_{3} \bar{\beta}_{4}+\alpha_{1} \bar{\alpha}_{3} \bar{\beta}_{4}\right)\left(\theta_{23}-\bar{\delta}_{2} \bar{\delta}_{3}\right)\left(\theta_{24}-\bar{\chi}_{2} \bar{\chi}_{4}\right) .
\end{aligned}
$$

The angular terms for operator 13 are

$$
\begin{aligned}
& \mathcal{F} \frac{13}{(1)}=\left(\theta_{14}-\bar{\alpha}_{1} \bar{\alpha}_{4}-\bar{\gamma}_{1} \bar{\gamma}_{4}+\alpha_{2} \bar{\alpha}_{1} \bar{\gamma}_{4}\right)\left(\theta_{13}-\bar{\beta}_{1} \bar{\beta}_{3}\right)\left(\theta_{34}-\bar{\lambda}_{3} \bar{\lambda}_{4}\right) \\
& \mathcal{F} \frac{13}{(2)}=\left(\theta_{34}-\bar{\epsilon}_{3} \bar{\epsilon}_{4}-\bar{\lambda}_{3} \bar{\lambda}_{4}+\epsilon_{6} \bar{\epsilon}_{4} \bar{\lambda}_{3}\right)\left(\theta_{13}-\bar{\beta}_{1} \bar{\beta}_{3}\right)\left(\theta_{14}-\bar{\alpha}_{1} \bar{\alpha}_{4}\right) \\
& \mathcal{F} \frac{13}{(3)}=\left(\theta_{14}-\bar{\beta}_{1} \bar{\beta}_{4}-\bar{\kappa}_{1} \bar{\kappa}_{4}+\beta_{5} \bar{\beta}_{1} \bar{\kappa}_{4}\right)\left(\theta_{13}-\bar{\alpha}_{1} \bar{\alpha}_{3}\right)\left(\theta_{34}-\bar{\delta}_{3} \bar{\delta}_{4}\right) \\
& \mathcal{F} \frac{13}{(4)}=\left(\theta_{13}-\bar{\beta}_{1} \bar{\beta}_{3}-\bar{\kappa}_{1} \bar{\kappa}_{3}+\beta_{5} \bar{\beta}_{1} \bar{\kappa}_{3}\right)\left(\theta_{14}-\bar{\alpha}_{1} \bar{\alpha}_{4}\right)\left(\theta_{34}-\bar{\epsilon}_{3} \bar{\epsilon}_{4}\right) \\
& \mathcal{F} \frac{13}{(5)}=\left(\theta_{13}-\bar{\alpha}_{1} \bar{\alpha}_{3}-\bar{\gamma}_{1} \bar{\gamma}_{3}+\alpha_{2} \bar{\alpha}_{1} \bar{\gamma}_{3}\right)\left(\theta_{14}-\bar{\beta}_{1} \bar{\beta}_{4}\right)\left(\theta_{34}-\bar{\chi}_{3} \bar{\chi}_{4}\right) \\
& \mathcal{F}-\frac{13}{(6)}=\left(\theta_{34}-\bar{\delta}_{3} \bar{\delta}_{4}-\bar{\chi}_{3} \bar{\chi}_{4}+\delta_{7} \bar{\delta}_{3} \bar{\chi}_{4}\right)\left(\theta_{13}-\bar{\alpha}_{1} \bar{\alpha}_{3}\right)\left(\theta_{14}-\bar{\beta}_{1} \bar{\beta}_{4}\right) .
\end{aligned}
$$

The angular terms for operator 14 are

$$
\begin{aligned}
& \mathcal{F} \frac{14}{(1)}=\left(\theta_{14}-\bar{\beta}_{1} \bar{\beta}_{4}-\bar{\lambda}_{1} \bar{\lambda}_{4}+\beta_{6} \bar{\beta}_{1} \bar{\lambda}_{4}\right)\left(\theta_{12}-\bar{\alpha}_{1} \bar{\alpha}_{2}\right)\left(\theta_{24}-\bar{\gamma}_{2} \bar{\gamma}_{4}\right) \\
& \mathcal{F} \frac{14}{(2)}=\left(\theta_{12}-\bar{\beta}_{1} \bar{\beta}_{2}-\bar{\lambda}_{1} \bar{\lambda}_{2}+\beta_{6} \bar{\beta}_{1} \bar{\lambda}_{2}\right)\left(\theta_{14}-\bar{\alpha}_{1} \bar{\alpha}_{4}\right)\left(\theta_{24}-\bar{\epsilon}_{2} \bar{\epsilon}_{4}\right) \\
& \mathcal{F} \frac{14}{(3)}=\left(\theta_{14}-\bar{\alpha}_{1} \bar{\alpha}_{4}-\bar{\delta}_{1} \bar{\delta}_{4}+\alpha_{3} \bar{\alpha}_{1} \bar{\delta}_{4}\right)\left(\theta_{12}-\bar{\beta}_{1} \bar{\beta}_{2}\right)\left(\theta_{24}-\bar{\kappa}_{2} \bar{\kappa}_{4}\right) \\
& \mathcal{F} \frac{14}{(4)}=\left(\theta_{24}-\bar{\epsilon}_{2} \bar{\epsilon}_{4}-\bar{\kappa}_{2} \bar{\kappa}_{4}+\epsilon_{5} \bar{\epsilon}_{4} \bar{\kappa}_{2}\right)\left(\theta_{12}-\bar{\beta}_{1} \bar{\beta}_{2}\right)\left(\theta_{14}-\bar{\alpha}_{1} \bar{\alpha}_{4}\right) \\
& \mathcal{F} \frac{14}{(5)}=\left(\theta_{24}-\bar{\gamma}_{2} \bar{\gamma}_{4}-\bar{\chi}_{2} \bar{\chi}_{4}+\gamma_{7} \bar{\gamma}_{2} \bar{\chi}_{4}\right)\left(\theta_{12}-\bar{\alpha}_{1} \bar{\alpha}_{2}\right)\left(\theta_{14}-\bar{\beta}_{1} \bar{\beta}_{4}\right) \\
& \mathcal{F} \frac{14}{(6)}=\left(\theta_{12}-\bar{\alpha}_{1} \bar{\alpha}_{2}-\bar{\delta}_{1} \bar{\delta}_{2}+\alpha_{3} \bar{\alpha}_{1} \bar{\delta}_{2}\right)\left(\theta_{14}-\bar{\beta}_{1} \bar{\beta}_{4}\right)\left(\theta_{24}-\bar{\chi}_{2} \bar{\chi}_{4}\right) .
\end{aligned}
$$

The angular terms for operator 15 are 


$$
\begin{aligned}
& \mathcal{F} \frac{15}{(1)}=\left(\theta_{23}-\bar{\gamma}_{2} \bar{\gamma}_{3}-\bar{\lambda}_{2} \bar{\lambda}_{3}+\gamma_{6} \bar{\gamma}_{2} \bar{\lambda}_{4}\right)\left(\theta_{12}-\bar{\alpha}_{1} \bar{\alpha}_{2}\right)\left(\theta_{13}-\bar{\beta}_{1} \bar{\beta}_{3}\right) \\
& \mathcal{F} \frac{15}{(2)}=\left(\theta_{12}-\bar{\alpha}_{1} \bar{\alpha}_{2}-\bar{\epsilon}_{1} \bar{\epsilon}_{2}+\alpha_{4} \bar{\alpha}_{1} \bar{\epsilon}_{2}\right)\left(\theta_{13}-\bar{\beta}_{1} \bar{\beta}_{3}\right)\left(\theta_{23}-\bar{\lambda}_{2} \bar{\lambda}_{3}\right) \\
& \mathcal{F} \frac{15}{(3)}=\left(\theta_{23}-\bar{\delta}_{2} \bar{\delta}_{3}-\bar{\kappa}_{2} \bar{\kappa}_{3}+\delta_{5} \bar{\delta}_{3} \bar{\kappa}_{2}\right)\left(\theta_{12}-\bar{\beta}_{1} \bar{\beta}_{2}\right)\left(\theta_{13}-\bar{\alpha}_{1} \bar{\alpha}_{3}\right) \\
& \mathcal{F} \frac{15}{(4)}=\left(\theta_{13}-\bar{\alpha}_{1} \bar{\alpha}_{3}-\bar{\epsilon}_{1} \bar{\epsilon}_{3}+\alpha_{4} \bar{\alpha}_{1} \bar{\epsilon}_{3}\right)\left(\theta_{12}-\bar{\beta}_{1} \bar{\beta}_{2}\right)\left(\theta_{23}-\bar{\kappa}_{2} \bar{\kappa}_{3}\right) \\
& \mathcal{F} \frac{15}{(5)}=\left(\theta_{13}-\bar{\beta}_{1} \bar{\beta}_{3}-\bar{\chi}_{1} \bar{\chi}_{3}+\beta_{7} \bar{\beta}_{1} \bar{\chi}_{3}\right)\left(\theta_{12}-\bar{\alpha}_{1} \bar{\alpha}_{2}\right)\left(\theta_{23}-\bar{\gamma}_{2} \bar{\gamma}_{3}\right) \\
& \mathcal{F} \frac{15}{(6)}=\left(\theta_{12}-\bar{\beta}_{1} \bar{\beta}_{2}-\bar{\chi}_{1} \bar{\chi}_{2}+\beta_{7} \bar{\beta}_{1} \bar{\chi}_{2}\right)\left(\theta_{13}-\bar{\alpha}_{1} \bar{\alpha}_{3}\right)\left(\theta_{23}-\bar{\delta}_{2} \bar{\delta}_{3}\right) .
\end{aligned}
$$

Finally, the angular terms for operator 16 are

$$
\begin{aligned}
& \mathcal{F} \frac{16}{(1)}=\left(\theta_{12}-\bar{\alpha}_{1} \bar{\alpha}_{2}\right)\left(\theta_{13}-\bar{\beta}_{1} \bar{\beta}_{3}\right)\left(\theta_{24}-\bar{\gamma}_{2} \bar{\gamma}_{4}\right)\left(\theta_{34}-\bar{\lambda}_{3} \bar{\lambda}_{4}\right)
\end{aligned}
$$

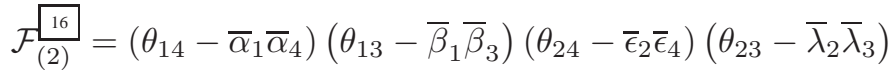

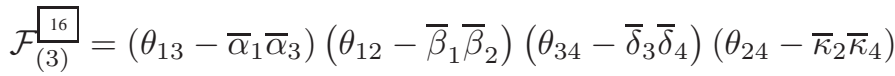

$$
\begin{aligned}
& \mathcal{F} \frac{16}{(4)}=\left(\theta_{14}-\bar{\alpha}_{1} \bar{\alpha}_{4}\right)\left(\theta_{12}-\bar{\beta}_{1} \bar{\beta}_{2}\right)\left(\theta_{34}-\bar{\epsilon}_{3} \bar{\epsilon}_{4}\right)\left(\theta_{23}-\bar{\kappa}_{2} \bar{\kappa}_{3}\right) \\
& \mathcal{F} \frac{16}{(5)}=\left(\theta_{12}-\bar{\alpha}_{1} \bar{\alpha}_{2}\right)\left(\theta_{14}-\bar{\beta}_{1} \bar{\beta}_{4}\right)\left(\theta_{23}-\bar{\gamma}_{2} \bar{\gamma}_{3}\right)\left(\theta_{34}-\bar{\chi}_{3} \bar{\chi}_{4}\right) \\
& \mathcal{F} \frac{\sqrt[16]{(6)}}{(6)}=\left(\theta_{13}-\bar{\alpha}_{1} \bar{\alpha}_{3}\right)\left(\theta_{14}-\bar{\beta}_{1} \bar{\beta}_{4}\right)\left(\theta_{23}-\bar{\delta}_{2} \bar{\delta}_{3}\right)\left(\theta_{24}-\bar{\chi}_{2} \bar{\chi}_{4}\right) \text {. }
\end{aligned}
$$

[1] R. Beck and R. Wielebinski, 'Magnetic Fields in Galaxies', Planets, Stars and Stellar Systems, Vol. 5: Galactic Structure and Stellar Populations, ed. G. Gilmore, Springer, Berlin 2013, ISBN 978-90-481-8817-8 arXiv:1302.5663v3 [astro-ph.GA]

[2] R. Beck, Astrophys. Space Sci. Trans. 5, 43 (2009).

[3] C. Vogt, T.A. Enßlin, Astron. Astrophys. 434, 67 (2005).

[4] T.E. Clarke, P.P. Kronberg, H. Bohringer, Astrophys. J. Lett. 547, L111 (2001).

[5] R. Durrer and A. Neronov, arXiv:1303.7121 v2 [astro-ph.CO].

[6] A. Brandenburg and K. Subramanian, Phys. Rep. 417, 1 (2005).

[7] R. M. Kulsrud and E. G. Zweibel, Rep. Prog. Phys. 71, 4, 046901 (2008).

[8] L. M. Widrow, Rev. Mod. Phys. 74, 775 (2002).

[9] M. L. Bernet, F. Miniati, S. J. Lilly, P. P. Kronberg and M. Dessauges-Zavadsky, Nature 454, 7202, 302 (2008).

[10] P. P. Kronberg, M. L. Bernet, F. Miniati, S. J. Lilly, M. B. Short, D. M. Higdon, Astrophys. J. 676, 70 (2008).

[11] A. Neronov and I Vovk, Science 328, 73 (2010).

[12] F. Tavecchio, G. Ghisellini, L. Foschini, G. Bonnoli, G. Ghirlanda and P. Coppi Mon. Not. R Astron. Soc., 406, L70L74 (2010).

[13] K.Dolag, M.Kachelriess, S.Ostapchenko and R.Tomas, Astrophys. J. Lett. 727, L4 (2011).

[14] K. Subramanian, D. Narasimha and S. M. Chitre, Mon. Not. R. Astron. Soc. 271, L15 (1994).

[15] R. M. Kulsrud, R. Cen, J. P. Ostriker and D. Ryu, Astrophys. J. 480, 481 (1997).

[16] N. Y. Gnedin, A. Ferrara and E. G. Zweibel, Astrophys. J. 539,
505 (2000).

[17] R. Gopal and S. K. Sethi, Mon. Not. R. Astron. Soc. 363, 521 (2005).

[18] M. S. Turner, L. M. Widrow, Phys. Rev. D 37, 2743 (1988);

[19] B. Ratra, Astrophys. J. 391, L1 (1992);

[20] J. Martin and J. Yokoyama, J. Cosmol. Astropart. Phys. 01, 025 (2008);

[21] M. Giovannini, Lect. Notes Phys. 737, 863 (2008);

[22] K. Subramanian, Astron. Nachr. 331, 1, 110 (2010);

[23] A. Kandus, K. E. Kunze and C. G. Tsagas, Phys. Rep. 505, 1 (2011).

[24] T. Vachaspati, Phys. Lett. B 265, 258 (1991).

[25] R. Banerjee and K. Jedamzik, Phys. Rev. D 70, 123003 (2004).

[26] A. Diaz-Gil, J. Garcia-Bellido, M. G. Perez and A. GonzalezArroyo, Phys. Rev. Lett. 100, 241301 (2008).

[27] C. J. Copi, F. Ferrer, T. Vachaspati and A. Achucarro, Phys. Rev. Lett. 101, 171302 (2008).

[28] T. Kahniashvili, A. Brandenburg, A. G. Tevzadze, and B. Ratra, Phys. Rev. D 81, 123002 (2010).

[29] P. A. R. Ade et al. [Planck collaboration XVI], arXiv:1303.5076 2 [astro-ph.CO].

[30] D. Paoletti and F. Finelli, Phys Lett. B 72645 (2013).

[31] D. Paoletti and F. Finelli, Phys. Rev. D 83, 123533 (2011).

[32] D. G. Yamazaki, K. Ichiki, T. Kajino and G. J. Mathews, Phys. Rev. D 81, 023008 (2010).

[33] J. R. Shaw and A. Lewis, Phys. Rev. D 86, 043510 (2012).

[34] M. Giovannini and K. E. Kunze, Phys. Rev. D 77, 063003 (2008). 
[35] K. Subramanian, Astron. Nachr. 327, 403 (2006).

[36] R. Durrer, New Astron. Rev. 51, 275 (2007).

[37] A. Kosowsky and A. Loeb, Astrophys. J. 469, 1 (1996);

[38] T. Kahniashvili, A. G. Tevzadze, S. K. Sethi, K. Pande and B. Ratra, Phys. Rev. D 82, 3005 (2010).

[39] L. Pogosian, A. P. S. Yadav, Y-F. Ng and T. Vachaspati, Phys. Rev. D 84, 3530 (2011).

[40] L. Verde, L.-M. Wang, A. Heavens and M. Kamionkowski, Mon. Not. R. Astron. Soc. 313, L141 (2000).

[41] J. Maldacena, Journal of High Energy Physics 5, 13 (2003);

[42] N. Bartolo, E. Komatsu, S. Matarrese and A. Riotto, Phys. Rep. 402, 103 (2004);

[43] E. Komatsu, Classical Quantum Gravity 27, 124010 (2010).

[44] P. Creminelli and M. Zaldarriaga, J. Cosmol. Astropart. Phys. 10, 006 (2004);

[45] D. Seery and J. E. Lidsey, J. Cosmol. Astropart. Phys. 06, 003 (2005);

[46] D. H. Lyth and Y. Rodriguez, Phys Rev. Lett. 95, 121302 (2005);

[47] C. T. Byrnes, M. Sasaki and D. Wands, Phys Rev. D 74, 123519 (2006).

[48] E. Komatsu and D. N. Spergel, Phys. Rev. D 63, 063002 (2001).

[49] T. R. Seshadri and K. Subramanian, Phys. Rev. Lett. 103, 081303 (2009).

[50] C. Caprini, F. Finelli, D. Paoletti and A. Riotto, J. Cosmol. Astropart. Phys. 06, 021 (2009).

[51] R.-G. Cai, B. Hu and H.-B. Zhang, J. Cosmol. Astropart. Phys. 08, 025 (2010).

[52] M. Shiraishi, D. Nitta, S. Yokoyama, K. Ichiki and K. Takahashi, Phys. Rev. D 82121302 (2010).

[53] M. Shiraishi, D. Nitta, S. Yokoyama, K. Ichiki and K. Takahashi, Phys. Rev. D 83123003 (2011).

[54] M. Shiraishi, D. Nitta, S. Yokoyama, and K. Ichiki, JCAP 03, 041 (2012).

[55] M. Shiraishi and T. Sekiguchi, arXiv:1304.7277 1 [astroph.CO].

[56] M. Shiraishi, J. Cosmol. Astropart. Phys. 11, 006 (2013).

[57] I. Brown, and R. Crittenden, Phys. Rev. D 72, 063002 (2005);

[58] I. A. Brown, Astrophys. J. 733, 83 (2011);

[59] P. Trivedi, K. Subramanian and T. R. Seshadri, Phys. Rev. D 82, 123006 (2010)

[60] P. Trivedi, T. R. Seshadri and K. Subramanian, Phys. Rev. Lett. 108, 231301 (2012)

[61] P. A. R. Ade et al. [Planck Collaboration XXIV], arXiv:1303.5084v2 [astro-ph.CO].

[62] C. Bonvin, C. Caprini and R. Durrer, Phys. Rev. D 88, 083515 (2013).
[63] K. Jedamzik, V. Katalinic and A. V. Olinto, Phys. Rev. D 57, 3264 (1998);

[64] K. Subramanian and J. D. Barrow, Phys. Rev. D 58083502 (1998).

[65] R. Durrer and C. Caprini, J. Cosmol. Astropart. Phys. 11, 010 (2003).

[66] M. Giovannini, PMC Physics A, 1:5 (2007) (doi:10.1186/17540410-1-5).

[67] F. Finelli, F. Paci and D. Paoletti, Phys. Rev. D 78, 023510 (2008)

[68] C. Bonvin and C. Caprini, J. Cosmol. Astropart. Phys. 05, 022 (2010).

[69] T. R. Seshadri and K. Subramanian, Phys. Rev. Lett. 87, 101301 (2001);

[70] A. Mack, T. Kahniashvili and A. Kosowsky, Phys. Rev. D 65, 123004 (2002);

[71] K. Subramanian, T. R. Seshadri and J. D. Barrow, Mon. Not. Roy. Astr. Soc. 344, L31 (2003).

[72] J. R. Shaw and A. Lewis, Phys. Rev. D 81, 043517 (2010).

[73] T. Okamoto and W. Hu, Phys. Rev. D 66063008 (2002).

[74] N. Kogo and E. Komatsu, Phys. Rev. D 73083007 (2006).

[75] J. R. Fergusson and E. P. S. Shellard, Phys. Rev. D 76, 083523 (2007).

[76] D.M. Regan, E.P.S. Shellard, J.R. Fergusson, Phys. Rev. D. 82 $023520(2010)$

[77] D. A. Varshalovich, A. N. Moskalev and V. K. Khersonskii, Quantum Theory of Angular Momentum (World Scientific Pub. Co. Inc. 1988).

[78] W. Hu, Phys. Rev. D 64083005 (2001).

[79] I. S. Gradshteyn and I. M. Ryzhik, Table of Integrals, Series and Products (Academic Press, New York, U.S.A. and London U.K., 6th edition, 2000).

[80] M. Zaldarriaga and U. Seljak, Phys. Rev. D 55, 1830 (1997);

[81] W. Hu, Phys. Rev. D 62, 043007 (2000)

[82] L. Boubekeur, P. Creminelli, G. DAmico, J. Norena, and F. Vernizzi, J. Cosmol. Astropart. Phys. 08029 (2009);

[83] A. Lewis, J. Cosmol. Astropart. Phys. 06023 (2012).

[84] M. Hindmarsh, C. Ringeval and T. Suyama, Phys. Rev. D 81, 063505 (2010).

[85] A. Lewis, J. Cosmol. Astropart. Phys. 10026 (2011).

[86] J. Smidt, A. Amblard, C. T. Byrnes, A. Cooray, A. Heavens, and D. Munshi Phys. Rev. D 81123007 (2010).

[87] E. Komatsu et al., Astrophys. J. Suppl. Ser. 180, 330 (2009).

[88] E. Komatsu et al., Astrophys. J. Suppl. Ser. 192, 18 (2011).

[89] C. Bonvin, C. Caprini and R Durrer, Phys. Rev. D 86, 023519 (2012). 